City University of New York (CUNY)

CUNY Academic Works

9-2015

\title{
Emotion Dysregulation: A Predictor For Cbt Treatment Outcomes In A Comorbid Ptsd And Sud Population
}

Jennifer Robin Wallach

Graduate Center, City University of New York

\section{How does access to this work benefit you? Let us know!}

More information about this work at: https://academicworks.cuny.edu/gc_etds/1178

Discover additional works at: https://academicworks.cuny.edu

This work is made publicly available by the City University of New York (CUNY).

Contact: AcademicWorks@cuny.edu 


\title{
EMOTION DYSREGULATION: A PREDICTOR FOR CBT TREATMENT OUTCOMES IN A COMORBID PTSD AND SUD POPULATION
}

\author{
by
}

Jennifer R. Wallach, M.P.H.

A dissertation submitted to the Graduate Faculty in Clinical Psychology in partial fulfillment of the requirements for the degree of Doctor of Philosophy, The City University of New York 
(C) 2015

Jennifer Robin Wallach

All Rights Reserved 
This manuscript has been read and accepted for the Graduate Faculty in Clinical Psychology in satisfaction of the dissertation requirement for the degree of Doctor of Philosophy.

Date

Denise Hien, Ph.D.

Chair of Examining Committee

Date

Joshua Brumberg, Ph.D.

Executive Officer, Psychology

(Print) Paul Wachtel, Ph.D.

(Print) Eric Fertuk, Ph.D.

(Print) Diana Punales, Ph.D.

(Print) Benjamin Harris, Ph.D.

Supervisory Committee 


\section{ABSTRACT \\ EMOTION DYSREGULATION: A PREDICTOR FOR CBT TREATMENT OUTCOMES IN A COMORBID PTSD AND SUD POPULATION}

By

\section{Jennifer Robin Wallach, M.P.H.}

Adviser: Denise Hien, Ph.D.

Trauma has been shown to disrupt self-regulatory processes; emotion regulation deficits have also been implicated in the initiation and maintenance of substance use disorders (SUDs).

Treatments for these complex comorbid problems utilize a variety of approaches. However, to date, few studies have examined the effect of various available treatments on emotion dysregulation in this population. This study extends the research on emotion regulation among the population with comorbid posttraumatic stress disorder (PTSD) and SUD. This study is a secondary analysis of part of the data from a randomized clinical trial assessing the relative efficacy of two active treatment conditions for substance dependent individuals who also meet criteria for current $D S M-I V$-TR PTSD. The current study examined features associated with emotion dysregulation, correlating dysregulation with PTSD and SUD symptoms; the study focused on baseline emotion dysregulation as a predictor of treatment outcomes. Additionally, the study examined emotion dysregulation over time to determine the mutability of this complex factor in response to treatment. Lastly, this study utilized a case illustration to examine in more depth change in emotion dysregulation over time, as measured by physiological arousal; this methodology provides for a more thorough depiction of the phenomenon. Correlation analyses identified that baseline emotion dysregulation was associated with baseline PTSD symptom severity, but not with baseline substance use frequency among those with comorbid PTSD and SUD. A repeated measures ANOVA determined that the high baseline dysregulation group that 
received relapse prevention treatment showed no change in substance use frequency from baseline to follow-up. Additionally, the high baseline dysregulation group that received Concurrent Treatment of PTSD and Substance Use Disorders Using Prolonged Exposure (COPE) showed a trending decrease in dysregulation at follow-up. Altogether, this exploratory study provided evidence that exposure therapy can be tolerated by and beneficial to highly dysregulated persons with both PTSD and SUD and that psychotherapy focusing only on substance used may be contraindicated for highly dysregulated individuals with comorbid PTSD and SUD.

Keywords: substance dependence, posttraumatic stress disorder, emotion dysregulation, prolonged exposure 


\section{ACKNOWLEDGEMENTS}

I would like to begin by expressing my respect and gratitude to the patients who participated in this project, courageously sharing their remarkable life stories. I wish them great luck in continuing to establish a feeling of both safety and freedom in the world.

I would also like to articulate thanks to my committee members for their help in making this dissertation a reality. I am especially grateful to Dr. Denise Hien, my mentor, adviser, supervisor, and chair, for her dedication, expertise, humility and ability to think clearly in times of chaos. I am also appreciative of Dr. Paul Wachtel for his consistent care, containment and support throughout my training. I am thankful for Dr. Eric Fertuck's involvement and interest in this project. I am grateful to Dr. Benjamin Harris and Dr. Diana Punales for their encouragement and for helping to cultivate my clinical instincts.

This study could not have been possible without the effort and attention of Dr. Teresa Lopez-Castro and Santiago Papini who facilitated the research project. I also owe gratitude to Dr. Sarai Bachelder for her diligence and expertise and for answering and re-answering all my statistical questions.

I am indebted to my parents, Carol and Ira Wallach, who taught me the importance of kindness and integrity. I am thankful for my sister, Rebecca, her unwavering loyalty and for our overlapping subjectivities. Thanks to Ilene for helping me to better understand and articulate complexities. Thank you to my friends and my dog, Marti, for infusing life with levity and support. Lastly, thank you to Sylvia Helfen, my grandmother, who taught me the importance of getting things done.

This dissertation is dedicated to Danny, my husband, whose shoulders have grown strong over the last decade, especially during the last 5 years while supporting me through this training. 


\section{TABLE OF CONTENTS}

ACKNOWLEDGEMENTS vi

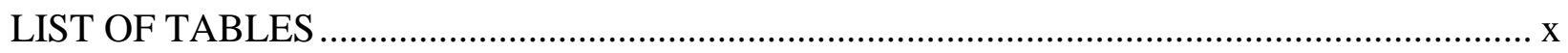

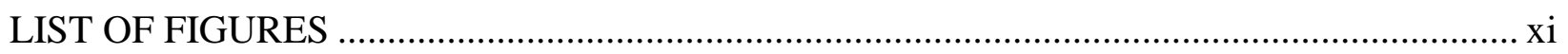

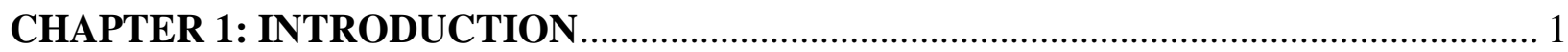

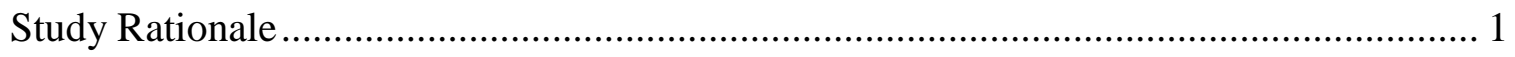

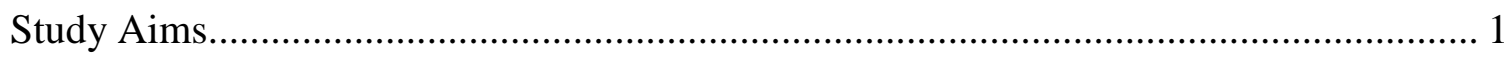

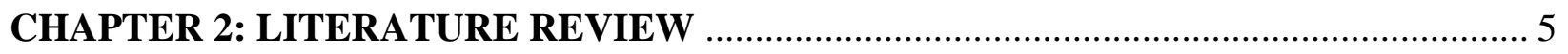

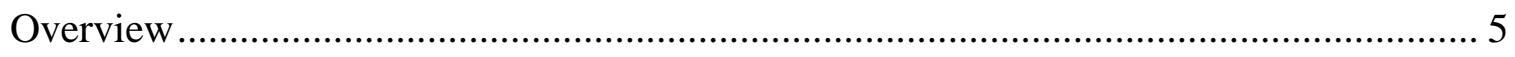

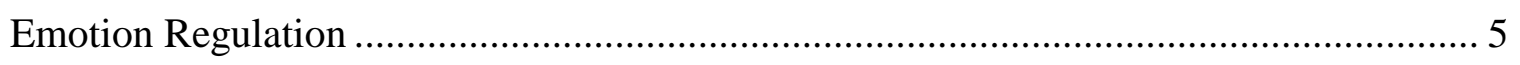

Developmental Conceptualization of Emotion Regulation ............................... 6

Neurobiological Response and Emotion Regulation .................................... 7

Cognitive-Behavioral Conceptualization of Emotion Regulation ........................ 9

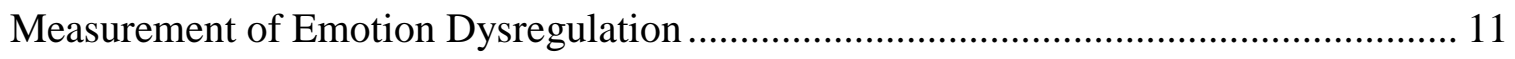

The Impact of Trauma Upon Emotion Regulation ................................................. 12

Emotion Dysregulation Among Substance Dependent Populations.............................. 14

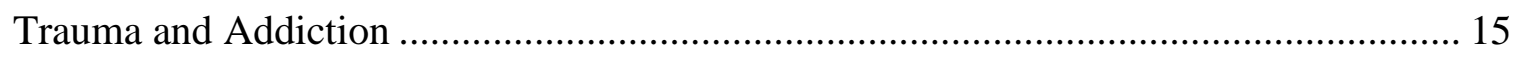

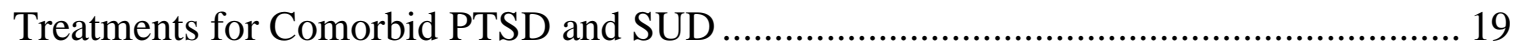

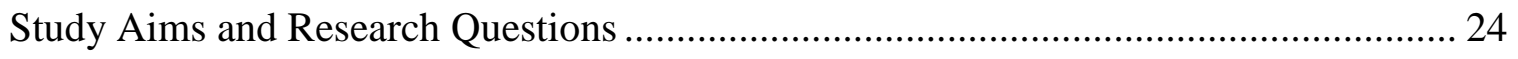

Rationale for Research Question 1 ....................................................... 25

Rationale for Research Question 2 ......................................................... 26

Rationale for Research Question 3 ........................................................ 26 


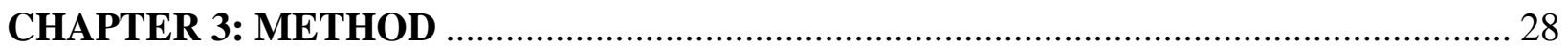

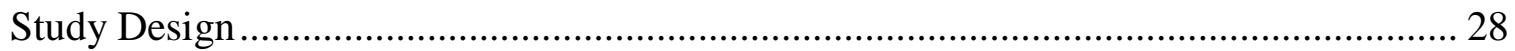

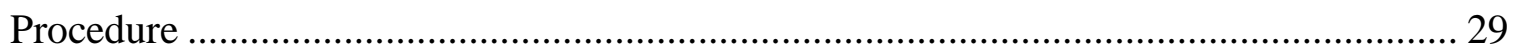

Sample

Behavioral Interventions ........................................................................... 30

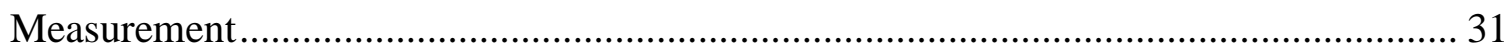

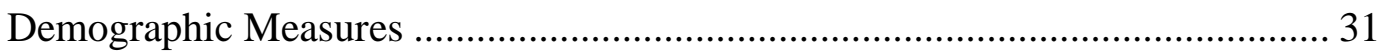

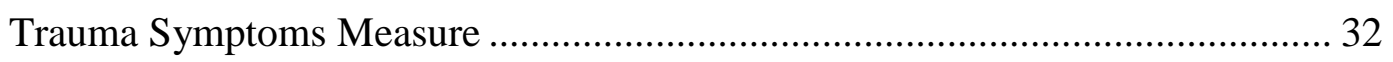

Substance Use Measure ............................................................................... 32

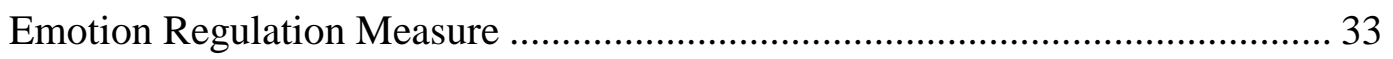

Physiological Arousal Measure .................................................................... 33

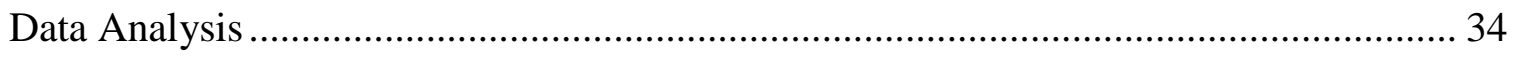

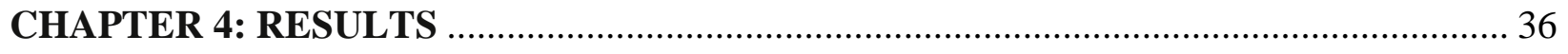

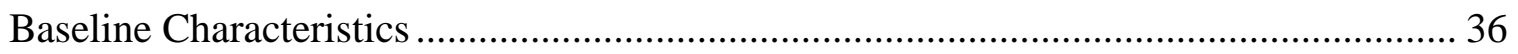

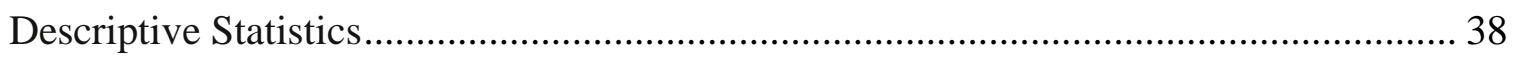

Research Question 1: Analysis .................................................................................. 38

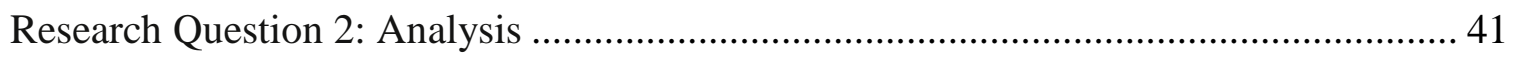

Research Question 3: Analysis ................................................................................. 46

Case Illustration .................................................................................................. 51

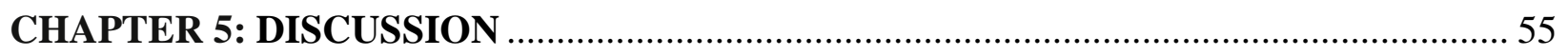

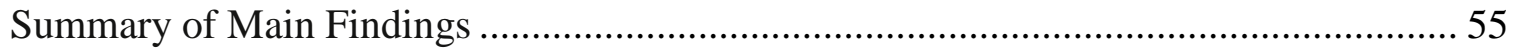

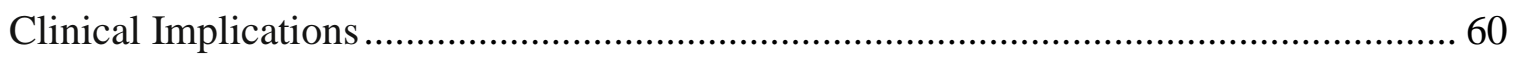

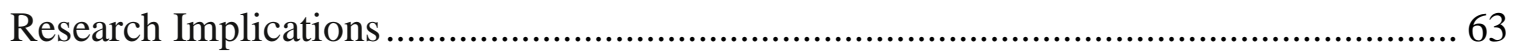




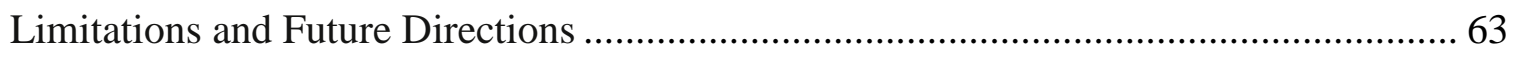

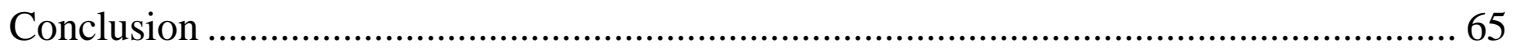

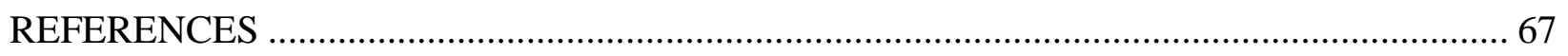




\section{LIST OF TABLES}

\section{Table}

1. Baseline Participant Characteristics 37

2. Descriptive Statistics: Substance Use, Emotion Dysregulation, and PTSD 39 Symptoms at Baseline

3. Correlation Analysis of Independent and Dependent Variables

4. Correlation Analysis Among Baseline DERS, Substance Use Days in the Last $7 \quad 41$ Days, and CAPS Scores $(N=80)$

5. Multivariate Tests: Change in Outcomes Over Time by Baseline DERS Group by 43 Treatment Group $(N=38)$

6. Univariate Tests for PTSD Symptoms by Time by Treatment Group by DERS Group $(N=38)$

7. Univariate Analysis for Substance Use Symptoms by Time by Treatment Group by DERS Group $(N=38)$

8. $t$ Tests for Baseline DERS Group by Treatment Group on CAPS Score, Regardless of Time

9. $t$ Tests for Time by Treatment Group by DERS Group on Substance Use

10. Univariate Tests for Change in DERS by Time by Treatment Group by DERS ( $N$ =38)

11. $t$ Tests for Time by DERS Group on Change in DERS

12. $t$ Tests for Time by Treatment Group by DERS Group on Change in DERS

13. $t$ Tests for Baseline DERS Group on Change in DERS, Regardless of Time

14. Case Illustration Participant Characteristics 


\section{LIST OF FIGURES}

\section{Figure}

1. Will Treatment Type Modify the Relationship Between Baseline Dysregulation, and Outcomes of Interest?

2. Baseline DERS Group by Treatment Group on CAPS Score 46

3. $t$ Tests for Time by Treatment Group by DERS Group on Substance Use 47

4. $\quad t$ Tests for Time by Treatment Group by DERS Group on Change in DERS $\quad 50$

5. Change in Physiological Arousal Through the Course of Treatment 53

6. Skin Conductance During First and Last COPE Session $\quad 54$ 


\section{CHAPTER 1: INTRODUCTION}

\section{Study Rationale}

The absence or impairment of emotion regulation abilities characterizes many psychological disorders of affect and thought. For instance, chronic worry and stress, avoidance or restriction of emotions, abrupt shifts in emotions and moods, and inappropriate affect, are among the symptoms of emotion dysregulation that define many clinical disorders (Cole, Michel, \& Teti, 1994). Research has implicated emotion dysregulation in posttraumatic stress disorder (PTSD) and substance use disorders (SUDs; Savov \& Atanassov, 2013; Schore, 2003). The present study examined symptoms and features associated with high baseline emotion dysregulation among a population with comorbid PTSD and SUD. Additionally, this research explored the role of emotion dysregulation for treatment outcomes. Lastly, the study assessed the mutability of emotion dysregulation among people with concurrent PTSD and SUD.

My interest in the area of emotion regulation arose from its critical role in adaptive functioning in stressful environments. The ability to understand and modulate affective responses to stress has been linked to emotional resilience and has been identified as a protective factor against pathology (Allen \& Fonagy, 2006). Examining the relationship between treatment and emotion dysregulation will help to inform best practices relevant to clinical populations struggling with deficits in the area of emotion regulation.

\section{Study Aims}

Emotion dysregulation is "inherent to many forms of psychopathology and may present in extremes of either avoidance and overly restricted emotional expression or heightened emotionality" (Bradley et al., 2011, p. 686). Emotion dysregulation is a multi-faceted construct involving a lack of awareness, understanding, and acceptance of emotions; an inability to control 
behaviors when experiencing emotional distress; a lack of access to adaptive strategies for modulating the intensity of emotional experiences; and an attempted avoidance of emotional distress (Gratz \& Roemer, 2004). Previous studies using non-clinical and community samples found associations between PTSD symptom severity and overall emotion dysregulation (Weiss, Tull, Anestis, \& Gratz, 2013). Similar associations were observed among a treatment-seeking cocaine dependent sample (McDermott, Tull, Gratz, Daughters, \& Lejuez, 2009). This study examined features associated with emotion dysregulation among a comorbid PTSD and SUD population. This research examined correlations between baseline emotion dysregulation and symptom severity (e.g., PTSD and substance dependence). It further sought to provide a more complete understanding of the relationship between emotion dysregulation and psychopathology among a comorbid PTSD and SUD population.

A comorbid PTSD and SUD diagnosis is associated with a range of negative clinical outcomes including high risk for treatment dropout (Brady, Dansky, Back, Foa, \& Carroll, 2001; Ford, Hawke, Alessi, Ledgerwood, \& Petry, 2007), relapse following SUD treatment (Hien, Nunes, Levin, \& Fraser, 2000; Najavits et al., 2007), more intense substance use patterns (Najavits et al., 2007), and high rates of high risk and self-destructive behaviors (Najavits et al., 2007). Despite these associations, few studies have examined psychological factors, such as emotion dysregulation, associated with these negative clinical outcomes. This study examined emotion dysregulation as a predictor for treatment outcomes.

Cognitive behavioral therapy (CBT) treatments are evidence-based interventions for PTSD and SUD (Foa, Keane, Friedman, \& Cohen, 2008; van Dam, Vedel, Ehring, \& Emmelkamp, 2012). Additionally, CBT has been shown to be effective for PTSD and SUD together (Najavits, 2002). Some CBT treatments for comorbid PTSD and SUD, like relapse 
prevention therapy (RPT), do not directly address trauma-related symptoms but instead focus on identifying high-risk use situations, bolstering coping strategies for managing relapse and cravings, finding ways to maintain lifestyle balance, and establishing more adaptive ways of responding to intense emotions. Other CBT treatments for comorbid PTSD and SUD, such as Concurrent Treatment of PTSD and Substance Dependence Using Prolonged Exposure (COPE), utilize an exposure technique to directly and experientially address emotion regulation problems and associated physiological arousal through habituation in order to disrupt fear responses. Some research suggests that this type of therapy may be helpful in the treatment of comorbid populations (Mills et al., 2012). Exposure therapy aims to help individuals assimilate and integrate their overwhelming experiences; addressing underlying dysregulation, which affects the intensity of the PTSD symptoms, may in turn reduce substance use. This relationship between PTSD and SUD is consistent with the concept of the self-medication hypothesis (Khantzian, 1997).

High levels of emotion dysregulation are common among individuals with comorbid PTSD and SUD. Clinicians are often hesitant to use exposure therapy with this population for fear that it is contraindicated and will lead to decompensation and/or dropout (van Minnen, Harned, Zoellner, \& Mills, 2012). Recent studies have challenged these assumptions and demonstrated the appropriateness of exposure therapy for treating comorbid PTSD and SUD (van Minnen et al., 2012). Patients in these studies did not get worse or demonstrate high rates of relapse; instead, they demonstrated improvements with regards to both substance use and PTSD outcomes (Brady et al., 2001; Mills et al., 2012; Najavits, Schmitz, Gotthardt, \& Weiss, 2005; Triffleman, Carroll, \& Kellogg, 1999). The extant research, however, is primarily limited to small pilot studies, with only one large randomized controlled trial completed to date. 
Additional research is necessary both to examine the extent to which treatment impacts emotion dysregulation among the comorbid PTSD and SUD population, and to determine the most effective and appropriate treatments for this population.

The present study, using pre- and post-self-report measures, examined the change in emotion regulation over time and explored the effects of baseline dysregulation on treatment outcomes based on treatment type. Lastly, exclusive reliance on self-report measures of emotion dysregulation is common among studies examining this psychological construct (e.g., Weiss et al., 2013). Self-report responses may be influenced by the participant's willingness or ability to report accurately on emotional responses. Accordingly, in addition to self-report, this study examined changes in emotion dysregulation over the course of a 12-week treatment using a physiological arousal measure. 


\section{CHAPTER 2: LITERATURE REVIEW}

\section{Overview}

The present study examined the relationship between treatment type and change in emotion regulation capacity in adults with comorbid PTSD and SUD, examining the effect of baseline dysregulation on treatment outcomes. A literature review is presented in the next three sections, beginning with an overview of emotion regulation, briefly summarizing developmental and cognitive theoretical conceptualizations, and reviewing physiological mechanisms underpinning emotion regulation and stress response. Additionally, emotion dysregulation among traumatized and substance dependent populations respectively is discussed. Next, the relationship between trauma and addiction is summarized, addressing the prevalence of PTSD and SUD, and highlighting the pathways between PTSD and SUD. The literature on several treatments that have been employed to effectively treat this comorbidity, such RPT and exposure-based treatments, is summarized. Finally, literature on the use of exposure treatments with highly dysregulated populations is reviewed, and the connection between emotion dysregulation and acting-out behaviors is presented.

\section{Emotion Regulation}

Gross and Thompson (2007) aptly pointed out that the roots of contemporary research on emotion regulation reside in the early study of psychological defenses, psychological stress and coping, attachment theory, and emotion theory. Emotion regulation ${ }^{1}$ involves monitoring, evaluating, and modifying internal feeling states and emotion-related physiological processes (Eisenberg, Cumberland, \& Spinrad, 1998). Ideal emotion regulation skills allow one to respond

\footnotetext{
${ }^{1}$ This dissertation uses both the terms emotion regulation and emotion dysregulation. Emotion regulation is used when referring to the successful monitoring, evaluating, and modifying internal feeling states and emotion-related physiological processes. However, when emotion regulation is not successful, emotion dysregulation occurs, which is by definition considered to be a dysfunctional state. This study was primarily focused on the mutability and impact of emotion dysregulation.
} 
in flexible and appropriate ways (Cicchetti, Ganiban, \& Barnett, 1991; Snyder, Schrepferman, \& St. Peter, 1997). Emotion regulatory processes may be automatic or controlled, conscious or unconscious, and may dampen, intensify, or sustain emotions, depending on an individual's goals (Gross \& Thompson, 2007). Various theorists have postulated different appraisal steps used to assess internal or external situations; these appraisals modulate and give rise to emotional responses.

\section{Developmental Conceptualization of Emotion Regulation}

Given the growing view that emotion regulation is a relational process, it follows logically that its early development occurs in the attachment relationship (Fonagy, Gergely, Jurist, \& Target, 2002). Schore (2003) underscored that it is within the attachment relationship that the primary caregiver shapes the development of the infant's coping responses. Although physiological, behavioral, and cognitive components clearly contribute to the emergence of emotion regulation skills, external factors — such as early interactions with caregivers — and direct methods of teaching children to behave in accordance with norms and expectations, also influence emotion regulation (Cicchetti et al., 1991). Consequently, emotion regulation can be viewed as an adaptive strategy that a child learns through interactions with the caregiving environment to maintain the attachment relationship (Main \& Solomon, 1990).

Describing it as instrumental in fostering the capacity for emotion regulation, Fonagy et al. (2002) discussed affect mirroring, which they defined as the parent's use of facial responses and vocalizations to represent an understanding of the infant's feeling states, and to be calming rather than intensifying. In addition, they suggested that self-reflection and the ability to reflect on the thoughts and feelings of others are capacities which are constructed, and have evolved from the mother-infant relationship. When caregivers do not contingently respond to the 
infant's affect, appropriate labeling of internal states does not occur, so that the affect remains both confusing and unsymbolized, and ultimately difficult to regulate (Calkins, 1994). Through repeated affect-mirroring experiences, the child begins to learn that his or her mental state is decoupled from external reality. Maladaptive early experiences with caregivers impact later development by limiting the ability to process or understand information related to mental states, which is critical to adaptive functioning in a stressful environment.

Tronick (1989) emphasized the importance of the caregiver's participation in interactive repair, which occurs when the infant's stress is regulated externally by dyadic exchanges. Schore (1994) asserted that these micro-regulations lead to structural changes in the infant over time. Schore (1994) referred to critical periods in the maturation of a child's emotional system, stating, "The outcome of effective dyadic affect regulatory transactions is an integration and restructuring of the infant's developing socioemotional system on a higher level of complexity" (p. 32). By providing well-modulated affective stimulation, the caregiver facilitates the growth of connections between cortical structures that neurobiologically mediate self-regulatory functions (Schore, 1994). Early object relational experiences, thus, directly influence the emergence of right-brain development and impact the ability to self-regulate emotional states (Schore, 1994). Regions in the prefrontal cortex mature gradually into adolescence; thus, there are periods in development when relational experiences organize reactivity and emotion regulation.

\section{Neurobiological Response and Emotion Regulation}

Given the growing view that self-regulatory capacities have neurobiological correlates, the next section of this review discusses the connection between neurobiological response and emotion regulation. The hypothalamic-pituitary-adrenal (HPA) axis is central to the task of 
emotion regulation and stress response (Herman, Ostrander, Mueller, \& Figueiredo, 2005). The hypothalamus, hippocampus, and regions in the prefrontal cortex all play significant roles in regulating the HPA axis. The HPA axis is a complex set of interactions between the hypothalamus, the pituitary gland, and the adrenal glands.

In response to an acute or chronic stressor, the hypothalamus, a small gland at the base of the brain serving as a regulatory center for body functions, releases corticotrophin-releasing factor (CRF) into the bloodstream. The CRF travels, via blood vessels, to the pituitary gland, a pea-sized structure located below the hypothalamus. In the anterior portion of the pituitary gland, CRF stimulates the release of adrenocorticotropic hormone (ACTH). ACTH, in turn, travels through the bloodstream from the pituitary gland to the two adrenal glands, located on top of each kidney (Southwick, Ozbay, Charney, \& McEwen, 2008). The outer portions of the adrenals glands are stimulated by ACTH to release other hormones called glucorticoids such as cortisol. The glucorticoids travel through the body as an adaptation to external stress. The rise in glucorticoids prompts a cascade of reactions in the human body, including a rush of energy and alertness. Glucorticoids like cortisol are also responsible for increasing blood sugar and breaking down proteins and fats to help mobilize energy (Southwick et al., 2008).

Glucorticoids play an integral role in a negative feedback system to control the HPA's reaction to stress. If the stressor is mild, when glucorticoids reach the hypothalamus, further excess release of CRF into the blood vessels is inhibited by hormones to a normal level. During times of intense stress, the hypothalamus signals for more CRF release; the inhibitory mechanism is overridden and the physiological stress reaction continues (Gunnar \& Fisher, 2006). 


\section{Cognitive-Behavioral Conceptualization of Emotion Regulation}

The limbic system, where the HPA axis is located, is the emotional center of the brain and is responsible for the identification, processing, and memory of a feeling state. It is tasked with determining next steps when an emotion is triggered (Hien, Litt, Cohen, Miele, \& Campbell, 2009). With this in mind, the next portion of this review will discuss cognitive aspects of emotion regulation.

Contemporary cognitive-behavioral conceptualizations of emotion regulation emphasize the role emotional arousal plays in adjusting decision-making processes, cueing behavioral responses, and enhancing or distorting memory (Gross \& Thompson, 2007). Emotions can be detrimental as well as helpful, depending upon their timing and intensity. Emotion regulation describes the processes by which individuals influence which emotions arise, when they arise, and how emotions are experienced and expressed (Hien et al., 2009). The ability to achieve goals, relate to others, and navigate life's complexities depends upon the ability to appropriately regulate emotions.

Emotion regulation was originally considered a demonstration of positive affect, but is now recognized to be a balance of both positive and negative affect. In addition, as the concept of emotion regulation has evolved over time, the importance of how much one regulates emotions has diminished, and the importance of both the range of accessible emotional responses and the degree to which a response appropriately matches a given situation has increased (Gross \& Munoz, 1995).

A subset of emotion regulation strategies is made up of the conscious cognitive regulation processes. Cognitive emotion regulation can generally be described as the management of emotionally activating or stressful information, with conscious cognitive 
strategies (Thompson, 1991). These cognitive processes are essential to the management of stressful events, enabling the individual to manage and attempt to gain control over emotional arousal (Zlomke \& Hahn, 2010). Gross and Thompson (2007) described emotion regulation as having five core processes: (1) situation selection, (2) situation modification, (3) attentional deployment, (4) cognitive change, and (5) response modulation. Situational selection and situational modification refer to both selecting and changing external situations to alter their emotional impact.

Attentional deployment refers to the way in which individuals direct their attention in a given situation to influence their emotions (Gross \& Thompson, 2007). Two major attentional deployment strategies are distraction and concentration. Distraction is the focusing of attention on different aspects of a situation, or moving attention away from a situation altogether in an effort to decrease arousal. Concentration is increasing the focus on the emotional features of a situation.

Emotion requires that percepts be imbued with meaning and that individuals evaluate their capacity to manage the situation (Gross \& Thompson, 2007). Cognitive change refers to altering how an individual perceives or appraises a situation, and adjusts the emotional significance by changing either how he or she thinks about the situation or about the capacity to manage the demands posed by the situation (Gross \& Thompson, 2007). Cognitive reappraisal, when used to down-regulate one's negative emotional response, refers to the reframing of a negative stimulus in less emotional terms (McRae, Ochsner, Mauss, Gabrieli, \& Gross, 2008). Response modulation refers to influencing physiological, experiential, or behavioral responses. Attempts to regulate the physiological and experiential aspects of emotion are 
frequently referred to as suppression and are a result of an individual's contextual assessment, as well as implicit and explicit social and societal demands (Gross \& Thompson, 2007).

In sum, research suggests that some combination of biological differences, environmental influences, social factors, and cognitive processes affect emotion regulatory capacities. By establishing an understanding of the mechanisms underlying differences in emotion regulatory functions, the interrelated nature of the factors and their influence on each other is clearer.

\section{Measurement of Emotion Dysregulation}

Emotion dysregulation has been described as a multi-faceted construct. In turn, many self-report instruments have been developed to measure various emotion regulation capacities (for a comprehensive list of measures see Aldao, Nolen-Hoeksema, \& Schweizemeta, 2010). The Difficulties in Emotion Regulation Scale (DERS; Gratz \& Roemer, 2004) represents the most comprehensive measure of the construct to date and it exhibited good reliability and validity in adults (Weinberg \& Klonsky, 2009). The DERS was designed to measure the complexities and clinically relevant difficulties of emotion regulation as described by several theories of emotion regulation (Sloan \& Kring, 2007).

On the one hand, because self-report measures are relatively easy and inexpensive to administer, they are commonly used in research settings. On the other hand, the extent to which individuals are able to accurately report their experiences of dysregulation has not been confirmed (Tamir, Robinson, \& Clore, 2002). The accuracy of self-report measures depends, at least partially, on the participant's insight, awareness, and capacity for metacognition (Cole, Martin, \& Dennis, 2004). Certain clinical populations, including traumatized populations, often have a diminished capacity to identify and label their emotional experiences which further complicates the utility of self-report measures. 
Alternatively, researchers have utilized observational methods to study emotion regulation, usually by instructing participants to engage in an emotion-eliciting stimulus and then observing the resultant emotions, cognitions, and physiological responses (e.g., NolenHoeksema, Wisco, \& Lyubomirsky, 2008). With increasing popularity, physiological measures of autonomic activity have been used as markers of emotional arousal. These measures of emotional arousal include electrodermal skin conductance, blood pressure, finger pulse, ocular startle reflex, pupil diameter, and heart rate (Bradley, Miccoli, Escrig, \& Lang, 2008; Gross, 2002). Specifically, the present study used a skin conductance measure to detect rapid fluctuations in sweat gland activity in response to the sympathetic nervous system (Boucsein, 1992); these fluctuations indicate changes in arousal and are markers for dysregulation. A case illustration utilizing skin conductance data will be presented.

\section{The Impact of Trauma Upon Emotion Regulation}

One of the components of emotion $d y s$ regulation is a tendency for emotions to quickly escalate and change rapidly, be expressed in unmodified forms, and overwhelm both coping capacity and reasoning (Bradley et al., 2011). Developmental research suggests that selfregulatory deficits result from an interaction of several factors, including temperamental and biological variables, as well as intrusive, chaotic, and stressful early life experiences, including abuse, neglect, and problematic attachment relationships (Schore, 1994, 2003). Emotion regulation skills allow for flexible and appropriate responses to stress and are essential to the development of many capacities, including optimal interpersonal functioning (Calkins, 1994; Cicchetti et al., 1991). Schore (2003) suggested that early life trauma, relational abuse, or dysregulated attachment systems can produce specific patterns of neurobiological alterations. 
Chronic early traumatic stress can disrupt maturing emotion regulation functions and increase the risk of lifelong deficits in this area (Teicher, 2000).

Some of the neurobiological alterations include the suboptimal functioning of the HPA axis. The literature posits that glucorticoids act through two types of receptors participating in the stress response. Type I maintains the capacity of the brain to respond to conditions that potentially threaten the organism. Type II counteracts the impact of the other stress processes and returns the organism to pre-stress levels of functioning (Gunnar \& Fisher, 2006). Normally, the feedback loop allows the body to disable these defenses when the threat passes. But in some cases, cortisol levels rise too often, or stay elevated too long (Gunnar \& Fisher, 2006). When the HPA axis is overactive, as in the case of chronic arousal, the negative feedback loop becomes progressively weaker (Gunnar \& Fisher, 2006). Repeated patterns of intense emotional experiences, like trauma exposure, may then lead to chronic changes in emotional sensitivity levels.

The HPA axis functions in concert with several other neural pathways. HPA dysfunctions are thought to likely to be associated with impaired integration of information from the amygdala, hippocampus, and prefrontal cortex (Herman et al., 2005). Research in this area seems to indicate that a suboptimally functioning HPA axis may be either hypo-responsive or hyper-responsive, affecting the functioning of the sympathetic and parasympathetic nervous systems (Gunnar \& Fisher, 2006). Although this body of research is still not well developed, the hypothesized links between childhood trauma and neurobiological consequences are thought to involve over-programming of neurotransmitters impacting the stress response. These irregular neurochemical responses are hypothesized to be detrimental to the brain during critical stages of development (Teicher, 2002). Neurobiological research on trauma and the stress response seem 
to evidence that neurobiological changes in major structures in the limbic system lead to dysregulation of arousal systems (Schore, 2003).

\section{Emotion Dysregulation Among Substance Dependent Populations}

Khantzian $(1997,2003)$ posited that substance users have difficulty tolerating negative emotions and managing overwhelming emotional states, and tend to use substances to achieve emotional stability. Khantzian and others have also asserted that substance abusers have not developed adaptive defensive mechanisms to deal with rage and aggression (Duncan, 1975; Glover, 1932; Khantzian, 2003; Radó, 1933). Aharonovich, Nguyen, and Nunes’s (2001) study examining levels of aggression and depression among individuals with SUDs, found higher levels of psychological distress in substance abusers than a non-clinical sample group. A study examining the capacity for emotion comprehension and emotion management found that individuals with SUDs scored lower on these dimensions than a non-clinical control group (Hertel, Schutz, \& Lammers, 2009). Hien and Miele (2003) found that emotion-focused coping style mediates the relationship between substance use and aggressive behavior. Together, these findings suggest that underlying emotion dysregulation contributes to an understanding of antisocial and aggressive behavior in this population. These results support the theoretical literature which suggests that individuals who abuse substances and exhibit aggressive behavior are likely to have emotion regulation deficits.

Emotion dysregulation and poor emotional coping skills have been identified as a risk factor for substance abuse disorders (Cooper, Frone, Russell, \& Mudar, 1995; Thorberg \& Lyvers, 2006). Additionally, a recent meta-analysis implicated high negative affect, low positive affect, and low effortful control in the initiation and maintenance of SUDs (Cheetham, Allen, Yucel, \& Lubman, 2010). Among individuals with SUDs, negative mood states have been 
shown to induce cravings (Childress et al., 1994). Research examining neurobiological substrates underlying SUDs highlight the dysregulation of brain reward and stress systems among this population (Cheetham et al., 2010; Koob, 2003). In turn, individuals may use drugs or alcohol in an attempt to lessen the biological effects of a dysregulated stress response system.

In sum, developmental research suggests that self-regulatory deficits result from an interaction of several factors, including temperamental and biological variables, as well as traumatic life experiences, including abuse, neglect, and problematic attachment relationships (Schore, 1994, 2003). Schore (2003) suggested that early life trauma, relational abuse, or dysregulated attachment systems can produce specific patterns of neurobiological alterations. Neurobiological research on trauma and the stress response suggest that neurobiological changes lead to dysregulation of arousal systems (Schore, 2003). Additionally, dysregulation and poor emotional coping skills have been identified as a risk factor for substance abuse disorders. Empirical and theoretical literature suggests that individuals who abuse substances and are likely to have emotion regulation deficits. In conclusion, emotional dysregulation is a shared feature of both trauma and addiction.

\section{Trauma and Addiction}

While comorbid individuals are often excluded from treatment research, all persons included in this study met criteria for both PTSD and SUD. The following section will present the diagnostic criteria relevant to the clinical population under investigation. Additionally, epidemiological data will be summarized, presenting the prevalence and incidence of SUD and PTSD in the population. Lastly, the pathways between SUD and PTSD will be presented and the hypothesized underlying mechanisms relating the two conditions will be discussed, highlighting the ways that emotional dysregulation is relevant to the co-occurring disorders. 
PTSD is a syndrome marked by the development of symptoms of intense horror or fear following a psychologically distressing event involving a real or perceived threat to physical well-being. This disorder typically results from an extreme, catastrophic, or overwhelming realworld experience and is typically accompanied by the following symptoms: re-experiencing the event through flashbacks, nightmares, and intrusive memories; avoidance of stimuli associated with the event; and increased arousal (American Psychiatric Association [APA], 2000).

The Diagnostic and Statistical Manual of Mental Disorders (4th ed., text rev.; DSM-IV$T R$; APA, 2000) provides diagnostic criteria for two forms of SUDs: substance abuse and substance dependence. Substance abuse is characterized by a maladaptive pattern of use of a substance leading to clinically significant impairment or distress. Maladaptive use is described as the repetition of at least one of the following: use in physically hazardous situations, substance-related legal problems, failure to fulfill major obligations, or interpersonal problems related to substance use. Substance dependence, too, is a maladaptive pattern of use that is characterized by three or more of the following conditions: increased tolerance of the substance; onset of withdrawal symptoms; using larger quantities of a substance, increased frequency of substance use or use for a longer period of time than was planned; unsuccessful efforts to reduce or regulate use; significant time spent procuring, using, or recuperating from use; disruption of important social, occupational, or recreational activities; and continued use despite recognizing its negative impact on a physical or psychological well-being (APA, 2000).

This study utilized the DSM-IV-TR for diagnostic purposes; the DSM-5 (APA, 2013), however, has since been published. The $D S M-5$ criteria for SUD are nearly identical to the $D S M-I V-T R$ substance abuse and dependence criteria combined into a single list. However, the threshold for a SUD diagnosis in DSM-5 is held at two or more criteria, in contrast to a threshold 
of one or more criteria for a diagnosis of DSM-IV-TR substance abuse and three or more for $D S M-I V$-TR substance dependence. Additionally, the PTSD diagnostic criteria have changed slightly. The stressor criterion (Criterion A) is more explicit about the experienced "traumatic" events. Furthermore, there were three major symptom clusters in DSM-IV-TR (re-experiencing, avoidance/numbing, and arousal). In the $D S M-5$, there are four symptom clusters; the avoidance/numbing cluster is divided into two distinct clusters: avoidance and persistent negative alterations in cognitions and mood. Because the criteria changes for these disorders were relatively minor, the findings from this study are likely generalizable to persons with comorbid PTSD and SUD as classified by the newer manual.

A few large-scale epidemiological studies have been conducted among the general population to assess the prevalence of PTSD and SUDs. One study indicated a 6.4\% lifetime prevalence of PTSD and a 35.3\% lifetime prevalence of SUDs (Kessler, Sonnega, Bromet, Hughes, \& Nelson, 1995). A second and more recent epidemiological study also estimated the lifetime prevalence of PTSD to be 6.4\% (Pietrzak, Goldtein, Southwick, \& Grant, 2011). Among individuals with PTSD, approximately $46 \%$ met criteria for SUD.

The prevalence of comorbid PTSD and SUD can be examined in several ways. One approach is to study the frequency of PTSD among those presenting with SUD, or conversely, the frequency of SUDs in those presenting with PTSD. Among treatment-seeking samples, rates of comorbid PTSD and SUDs are high. Individuals with PTSD were found to be up to 14 times more likely than patients without PTSD to have a SUD (Chilcoat \& Menard, 2003; Ford, Russo, $\&$ Mallon, 2007). According to current literature, prevalence estimates for PTSD in SUD samples vary from $11 \%$ to $41 \%$ (Harrington \& Newman, 2007; Ouimette, Goodwin, \& Brown, 2006; van Dam, Ehring, Vedel, \& Emmelkamp, 2010). Additionally, patients with comorbid 
SUD and PTSD show higher symptom severities and worse treatment outcomes compared to patients with either disorder alone (van Dam et al., 2012).

Although there is a well-established association between PTSD and SUD, the precise underlying mechanisms that relate the two conditions are unclear. Three pathways have been hypothesized. One, the high risk hypothesis, proposes that substance abuse leads to a higher risk for exposure to traumatic experiences, thereby increasing the opportunity for developing PTSD (Hien, Cohen, \& Campbell, 2005). A second, the susceptibility hypothesis, proposes that concurrent PTSD and SUD are due to an underlying, biological vulnerability, increasing the risk for developing both disorders independently following exposure to a trauma (Stewart \& Conrod, 2002). Lastly, the self-medication hypothesis, posits that individuals with trauma histories and related PTSD frequently use substances in an effort to manage or avoid distressing symptoms and to relieve painful emotions or physical sensations associated with their traumas (Khantzian, 1997; Stewart \& Conrod, 2002).

To date, the self-medication hypothesis is the only causal model with empirical support (Stewart \& Conrod, 2002). Chilcoat and Breslau (1998) conducted a study testing causal pathways between PTSD and SUD in a sample of 1,007 young adults. Using a prospective design and analytical strategies testing for causal inference, the study was able to expound upon findings from cross-sectional design studies. Support was only observed for only the selfmedication hypothesis.

Additionally, Hien, Jiang et al. (2010) conducted a study examining the temporal course of improvement in PTSD and SUD symptoms among women in outpatient substance abuse treatment. They found that PTSD symptom severity reductions were more likely to be associated with a reduction in substance use. Their analysis showed minimal evidence of 
substance use symptom reduction improving PTSD symptoms. Results support the selfmedication model, using substances to cope with PTSD symptoms.

Additionally, underlying emotion regulation deficits have been associated with the initiation and maintenance of SUD and may play an important mediating role between trauma exposure and subsequent substance abuse (Horowitz, Overton, Rosenstein, \& Steidl, 1992; Suh, Ruffins, Robins, Albanese, \& Khantzian, 2008). Addiction literature suggests that emotion regulation deficits, including low tolerance for painful feelings and inability to self-soothe, are typical of substance abusers (Cheetham et al., 2010). Krystal and Raskin (1970) proposed that because difficult emotions often remain somaticized, and unverbalized among substance abusers, people abusing substances are left ill-equipped to deal with their feelings. Self-medicating with alcohol and drugs can provide temporary relief from the hyperarousal and numbing symptoms, the dysregulated feeling states that are characteristic of PTSD (Hien et al., 2009). Though substance use may result in the short-term reduction of PTSD-related symptoms and attendant emotional distress, it is likely to have paradoxical consequences in the long term (Weiss et al., 2013). Substance use is theorized to prevent exposure to or awareness of corrective information and interfere with emotional processing (Foa \& Kozak, 1986). Thus, using substances to regulate emotions may exacerbate and prolong PTSD symptoms and emotion dysregulation in the long term, increasing motivations to use substances as an avoidant regulation strategy (Hayes, Wilson, Gifford, Follette, \& Strosahl, 1996).

\section{Treatments for Comorbid PTSD and SUD}

The developmental and cognitive theoretical conceptualizations as well as the physiological mechanisms underpinning emotion dysregulation have been outlined above. The ability to modify emotions in a desired direction and to accept and tolerate undesired emotions is 
requisite for optimal mental health. Emotion regulation deficits common among traumatized and substance dependent populations have been implicated in the initiation and maintenance of comorbid PTSD and SUD. Treatments for these complex comorbid problems utilize a variety of approaches. However, to date, few studies have examined the effect of the various available treatments on emotion dysregulation in this population. Additionally, there is a paucity of research which examines the effect of emotion dysregulation on treatment efficacy. The present study will examine correlations between baseline emotion dysregulation and symptom severity, establish emotion dysregulation as a predictor for treatment outcomes, examine the extent to which treatment impacts emotion dysregulation among a comorbid PTSD and SUD population, and examine changes in emotion dysregulation over the course treatment.

Clinical interventions for comorbid PTSD and SUD can be divided into two broad types: those which utilize prolonged exposure techniques (often also called present-focused or traumafocused) and those that do not. Exposure therapy, directly and experientially, addresses emotion dysregulation problems and associated physiological arousal through habituation to disrupted fear responses. The second type of treatment for comorbid PTSD and SUD focuses mainly on skills training, building coping strategies to manage overwhelming situations and emotions. While exposure therapy is currently considered one of the treatments of choice for PTSD alone (van Minnen et al., 2012), there is little consensus in the literature regarding the appropriateness of exposure therapy for highly dysregulated and comorbid PTSD and SUD populations. With an eye toward the role of emotion dysregulation, the next section of this literature review provides a summary of clinical interventions used to address dysregulation and symptomatology associated with the comorbid PTSD and SUD population. 
Skills training treatments include interventions such as Seeking Safety (Najavits, 2002), CBT for PTSD in SUD treatment (McGovern et al., 2009), Substance Dependence PTSD therapy (Triffleman, Carroll, \& Kellogg, 1999), Transcend (Donovan, Padin-Rivera, Kowaliw, 2001), and RPT (as described in Carroll, 1996). The skill-training treatments are divided into integrative treatments, those that focus on PTSD and SUD simultaneously, and sequential treatments, those that treat the disorders one at a time. The standard mode of care for comorbid PTSD and SUD in many substance abuse treatment programs is to treat the SUD first and to defer treatment of trauma-related issues. The clinical rationale behind deferring trauma treatment relates to a fear of that unaddressed substance abuse could impede other therapeutic efforts and potentially cause relapse (Pitman et al., 1991). The integrated model of treatment for comorbid PTSD and SUD requires that both disorders both be targeted simultaneously throughout the treatment. The integrated model posits that addressing trauma early in a treatment and providing concurrent relief form PTSD symptoms improves recovery from SUDs (Back, 2010; Hien, Campbell, Ruglass, Hu, \& Killeen, 2010).

Hien, Cohen, Miele, Litt, and Capstick (2004) compared two skills training CBT treatments, Seeking Safety, a gold standard integrative treatment for PTSD and SUD and RPT with comorbid PTSD and SUDs. RPT impacted both PTSD and SUD symptoms as effectively as Seeking Safety, the integrated treatment. It is hypothesized that the skills gained through RPT generalized to PTSD-related difficulties including, for example, issues of self-care, safety, depression, and anxiety without direct emphasis on trauma in the treatment. Overall, findings provided evidence that RPT is an efficacious, short-term treatment for comorbid PTSD and SUD symptoms. While it was not measured, Hien et al. (2004) suggested that both Seeking Safety 
and RPT may positively alter the emotion regulation capacities of individuals with comorbid PTSD and SUD.

Alternatively, several approaches to treatments for comorbid PTSD and SUD utilize a two-pronged strategy, employing both skill training and exposure-based therapy simultaneously. These types of interventions include Seeking Safety plus Exposure Therapy-Revised (Najavits et al., 2005), Concurrent Treatment of PTSD and Cocaine Dependence (Back, Dansky, Carroll, Foa, \& Brady, 2001) and COPE (Mills et al., 2012). Experts in the field of substance abuse treatment, however, have historically been hesitant to use prolonged exposure to treat individuals with comorbid PTSD and SUD. The exposure-based behavioral therapies, particularly those that involve imaginal re-experiencing of trauma, were suspected to be contraindicated for people with addictions, for fear of relapse, treatment dropout, increased acting-out behaviors, and dysregulation (van Minnen et al., 2012). In sum, prolonged exposure was considered inappropriate for use among patients with SUD as it was believed that these patients would be unable to cope with the intense emotions triggered during exposure, placing them at increased risk for relapse (Foa \& Rothbaum, 1998).

Acting-out behaviors can represent an attempt to cope with triggered or sustained posttraumatic emotional states, especially when these states overwhelm emotion regulation capacities (van der Kolk et al., 1996). Interpersonal trauma may especially activate acting-out behaviors because it produces heightened negative emotional states and disrupts the biological and psychological aspects of emotional regulation that ordinarily serve to regulate intense emotion (Pearlman \& Courtois, 2005). Briere, Hodge, and Godbout (2010) conducted a study examining the relationship between interpersonal trauma, PTSD, emotion dysregulation, and acting-out behaviors. Their findings suggested that level of PTSD symptomatology alone does 
not predict acting-out behaviors. Instead such behaviors are triggered and reinforced by reduced emotion regulation capacity (Briere et al., 2010). The relationship between acting-out behaviors and emotion regulation capacity reinforced concerns that PTSD treatments involving prolonged exposure may prove to be too activating for individuals who have a particular difficulty downregulating heightened negative emotional states.

Alternatively, a recent empirical study examining the impact of dysregulation on the efficacy of prolonged exposure suggested that patients presenting with severe dissociative symptoms profit similarly from exposure treatment as do patients with minimal dissociative symptoms (Hagenaars, van Minnen, \& Hoogduin, 2010). The findings from this study challenge the assumed contraindication of employing prolonged exposure with dysregulated populations.

Until recently, prolonged exposure was considered theoretically inappropriate for use among patients with substance dependence (van Minnen et al., 2012). It was widely believed that these dually diagnosed individuals would have difficulty coping with the intense emotional activation elicited by exposure therapy (Killeen, Back, \& Brady, 2011). Recently, the field's skepticism has begun to be empirically addressed (Mills et al., 2012). To date, existing research is primarily limited to smaller pilot studies (Brady et al., 2001; Coffey, Stasiewicz, Hughes, \& Brimo, 2006; Triffleman et al., 1999). Recently, however, one large randomized control trial evaluating the efficacy of prolonged exposure therapy for individuals with PTSD and SUD was conducted (Mills et al., 2012).

The Mills et al. (2012) randomized control trial $(N=103)$ compared COPE plus usual treatment with usual treatment for substance dependence. COPE consisted of 13 individual 90minute sessions with a clinical psychologist and usual treatment included any type of substance use treatment available in the community. From baseline to 9-month follow-up, the combined 
use of COPE plus usual treatment resulted in a significant improvement in PTSD symptom severity as compared to usual treatment. Both treatment groups demonstrated significant reductions in severity of substance dependence, substance use, depression, and anxiety; however, the difference between groups was not significant.

Despite exposure therapy's promise and significant efficacy in treating PTSD, the main clinical concern, which has likely limited the wider application of this techniques in comorbid PTSD and SUD populations, is tolerability. The Mills et al. (2012) COPE study revealed that exposure did not worsen patients' symptoms and in fact it significantly improved PTSD, substance use, and general psychiatric symptoms among treatment completers. Emotion dysregulation appears to be a fundamental aspect of both trauma and addiction. Treatments targeted to address this type of outcome have yet to examine (1) emotion dysregulation as a predictor for treatment outcomes, (2) the extent to which treatment impacts emotion dysregulation among a comorbid PTSD and SUD population, and 3) changes in emotion dysregulation over the course treatment. The present study will provide additional empirical data to further inform the field about safe and efficacious treatments for this difficult-to-treat population.

\section{Study Aims and Research Questions}

PTSD and SUD are frequently comorbid with prevalence estimates varying from 11 to 41\% (van Dam et al., 2010). Because comorbid PTSD and SUD is associated with poorer psychological treatment outcomes, and increased service utilization than either disorder alone, comorbid PTSD and SUD remains a serious public health concern. Given the high prevalence of PTSD and SUD comorbidity, it is important to further understand the underlying mechanisms of 
the disorders and to develop and evaluate treatment approaches appropriate for this vulnerable population.

Trauma has been shown to disrupt self-regulatory processes; emotion regulation deficits have also been implicated in the initiation and maintenance of SUDs (Hien et al., 2005). In sum, emotion dysregulation is a shared feature of both PTSD and SUD. Treatments for these complex comorbid problems utilize a variety of approaches. However, to date, few studies have examined the effect of the various available treatments on emotion dysregulation in this population.

In conclusion, this study aimed to extend the research on emotion dysregulation among the comorbid PTSD and SUD population. It examined features associated with emotion dysregulation, correlating dysregulation with myriad symptoms affecting this clinical population. Specifically, the study focused on baseline emotion dysregulation as a predictor of treatment outcomes. The overall study offered three exploratory research questions.

\section{Rationale for Research Question 1}

Emotion dysregulation is inherent to many forms of psychopathology (Bradley et al., 2011). It is a multifaceted construct involving a lack of awareness, understanding, and acceptance of emotions; an inability to control behaviors when experiencing distress; a lack of access to adaptive strategies for modulating the intensity of emotional experiences; and an attempted avoidance of emotional distress (Gratz \& Roemer, 2004). Previous studies using nonclinical and community samples found associations between PTSD symptom severity and overall emotion dysregulation (Weiss et al., 2013). Similar associations were observed among a treatment-seeking cocaine dependent sample (McDermott et al., 2009). This study will examine features associated with emotion dysregulation among a comorbid PTSD and SUD population. 
Specifically, the first research question will examine diagnostic features associated with emotion dysregulation at baseline.

Research Question 1. Will there be a relationship between baseline emotion dysregulation, as measured by the DERS, and baseline symptoms of PTSD and SUD, as measured by the Clinician-Administered PTSD Scale (CAPS) and Timeline Follow Back (TLFB)?

\section{Rationale for Research Question 2}

From a neurobiological perspective, several parts of the brain have been implicated in emotion regulation processes. In particular, structures in the limbic system have been shown to play a role in learned emotional associations that become automatic over time (i.e., bottom-up processes). Other structures in the brain, particularly areas located in the frontal lobes, have been implicated in the regulation of emotion (i.e., top-down processes). Exposure-focused treatments and skills-based treatments are premised on different understandings of emotion dysregulation. RPT aims to change behavior through a top-down approach, examining cognitions in relation to affect. COPE, the only exposure treatment developed to treat SUD, utilizes a bottom-up fear habituation processing approach. Specifically, the second research question explored whether baseline emotion dysregulation is a predictor for treatment outcomes.

Research Question 2. Will treatment type moderate the relationship between baseline dysregulation, as measured by the DERS, and treatment outcomes, as measured by the CAPS and substance use days in the last 7 at follow-up?

\section{Rationale for Research Question 3}

To the extent that certain emotion regulatory strategies are theorized to be more adaptive than others, it can be hypothesized that psychologically based treatments may affect emotion 
regulatory capacities. Empirical evidence that treatment, in fact, alters emotion regulation is currently sparse. Demonstrating how emotion regulation is changed by therapy is an important avenue for future research.

Because self-report measures are relatively easy and inexpensive to administer, they are commonly used in research settings. The accuracy of self-report measures depends, at least partially, on the participant's insight, awareness, and capacity for metacognition (Cole et al., 2004). Certain clinical populations, including traumatized populations, often have a diminished capacity to identify and label their emotional experiences which further complicates the usefulness of self-report measures.

Alternatively, researchers have used observational methods to study emotion regulation, usually by instructing participants to engage in an emotion-eliciting stimulus and then observing the resultant emotions, cognitions, and physiological responses. Despite compelling reasons to use multiple methods to assess emotion dysregulation, few studies have done so. This study however, used a skin conductance measure to detect rapid fluctuations in sweat gland activity in response to the release of acetylcholine by the sympathetic nervous system (Boucsein, 1992); these fluctuations indicate changes in arousal.

Specifically, the third research question examined change in emotion dysregulation preand post-intervention as well as over the course of treatment.

Research Question 3. Will treatment type moderate the relationship between baseline dysregulation, as measured by the DERS, and emotion dysregulation at follow-up, as measured by DERS total score at follow-up and physiological arousal over the course of a 12-week treatment? 


\section{CHAPTER 3: METHOD}

\section{Study Design}

This dissertation research is a secondary analysis of part of the data from a randomized clinical trial assessing the relative efficacy of two active treatment conditions for substance dependent individuals who also met criteria for current DSM-IV-TR PTSD. Eligible participants (see inclusion and exclusion criteria below) were randomly assigned to: (1) COPE, (2) RPT, or (3) delayed treatment. The study was a three-armed randomized controlled trial with repeated outcome measures at baseline; post treatment; and 1,2, and 3 months post-treatment. Trained therapists administered the behavioral interventions on an individual basis for 12 one-hour weekly sessions.

This $2 \times 2$ pre-post control group design study examined the correlation between baseline emotion dysregulation (as measured by the DERS, a self-report measure) and symptom severity at baseline (as measured by the Clinician-Administered PTSD Scale and the Timeline Follow Back). Secondly, the study examined baseline emotion dysregulation as a predictor for treatment outcomes (as measured by the Clinician-Administered PTSD Scale and the Timeline Follow Back) to identify differences in the predictive power of baseline dysregulation based on treatment type (i.e., RPT or COPE). Thirdly, the study assessed longitudinal changes in emotion dysregulation over time (at baseline and at 1 week post-treatment), examining a relationship between change in emotion dysregulation (as measured by the DERS) and treatment type (i.e., RPT or COPE). Lastly, we examined longitudinally changes in emotion dysregulation (as measured by physiological arousal) over the course of a 12-week treatment. 


\section{Procedure}

\section{Sample}

Individuals referred from the Addiction Institute of New York, and the Women's Health Project Treatment Research Center, as well as participants referred from other sources, including those responding to advertisements in the local papers and on Craigslist were screened. A total of 80 men and women were randomized into treatment conditions.

Eligible participants were between 18 and 65 years of age and able to comprehend English. All participants met DSM-IV-TR criteria for current drug or alcohol dependence and used illicit substances within the past 3 months. Participants also met DSM-IV-TR criteria for current PTSD in response to an interpersonal trauma. Participants all provided informed consent and understood all assessment instruments. Participants all provided the name of at least one person who would know where to contact them if they were to move or change phone numbers. Participants were willing to commit to 12 therapy sessions and five research assessment interviews.

Participants were deemed ineligible if they had a history of or current psychotic, schizoaffective, bipolar, eating, or dissociative identity disorder. These individuals were not considered psychiatrically stable enough to tolerate or benefit from the proposed treatment. Participants, however, with other psychiatric disorders, including mood or anxiety disorders, were included, due to the high frequency of comorbid psychiatric disorders among patients with PTSD (Brady, Killeen, Saladen, Dansky, \& Becker, 1994). Additionally, participants who presented a serious homicide or suicide risk or were involved in a violent intimate partnership (perpetrator or victim) were ineligible for the study. Participants already receiving PTSDspecific treatment or those on antidepressants, mood stabilizing medications, or methadone- 
initiated during the less than 8 weeks prior to study participation—were excluded from the study. Lastly, individuals with organic mental syndrome as indicated by a score of less than or equal to 21 on the Mini-Mental Status Exam were not eligible for the study.

\section{Behavioral Interventions}

\section{Cognitive-Behavioral Therapy in Conjunction with Prolonged Exposure (COPE).}

COPE is a manualized 12-week intervention, using cognitive-behavioral strategies and prolonged exposure techniques to decrease PTSD and SUD symptoms in individuals with concurrent PTSD and substance dependence. COPE represents a combination of empirically validated and efficacious cognitive-behavioral treatments for substance dependence and PTSD (Carroll, Nich, \& Rounsaville, 1998; Foa \& Rothbaum, 1998). In COPE, patients receive psychoeducation pertaining to the underlying principles of cognitive and exposure-based therapies in addition to the interrelatedness of PTSD and substance dependence. Coping skills training, relapse prevention techniques, and cognitive restructuring are taught to reduce substance use severity. In-vivo and imaginal exposure therapy techniques are used to reduce PTSD symptom severity. The primary goals of COPE are to (1) educate patients about the relationship between substance dependence and PTSD, (2) initiate and maintain abstinence, and (3) reduce PTSD symptom severity.

Relapse prevention treatment (RPT). RPT is a widely used cognitive-behavioral skills training designed to initiate and maintain abstinence from various types of substance (Carroll, Rounsaville, \& Gawin, 1991; Rawson, Obert, McCann, \& Marinelli-Casey, 1993). The RPT model is based on the concept that the same cognitive, behavioral, and affective mechanisms underlie all processes of relapse, regardless of the actual problem behavior (Marlatt \& Gordon, 
1985). By using RPT as a comparison intervention, it was possible to examine the specific effects of exposure therapy integrated into a CBT platform.

In general, RPT consists of the following elements: (1) psychoeducation about the biological, psychological, and social effects of addiction to and recovery from various substances; (2) identification of high-risk situations for and early signs of relapse; (3) development of coping skills for achieving abstinence-especially refusal and avoidance skills, behavioral skills for substituting drug use with drug-free behaviors in high risk situations, cognitive skills for overcoming cravings, drug-free stress management skills, and affective skills for labeling and expressing emotions without drugs; (4) increasing perceived self-efficacy to achieve and maintain abstinence; (5) development of cognitive and behavioral skills for preventing the abstinence violation effect; and (6) development of drug-free lifestyle behaviors.

\section{Measurement}

\section{Demographic Measures}

Demographic and Treatment History Form (DEMO). The DEMO is a structured 62item social and treatment history interview designed to provide basic demographic and life history information related to the course of illness, including: demographic variables, family history, age of onset of substance and/or psychiatric problems, history of hospitalizations, previous treatment, symptoms, and diagnoses (Hien \& Zimberg, 1991). The measure was administered five times: at baseline; immediately post-12-week intervention; and 1, 2, and 3 months post-treatment.

Any baseline variables including age, age of onset of psychiatric and SUDs, symptom severity, other $D S M-I V$-TR diagnoses, and trauma-related characteristics (e.g., type of trauma, 
age at which first trauma occurred) — which significantly differed across study arms—were treated as covariates and controlled for in the analyses.

\section{Trauma Symptoms Measure}

Clinician-Administered PTSD Scale (CAPS). The CAPS is a structured, clinical interview for assessing the cardinal and hypothesized frequency and intensity of signs and symptoms of PTSD. The CAPS measures DSM-IV-TR symptoms of PTSD, associated symptoms of PTSD, validity of responses, impairments in social and occupational functioning, and overall symptom severity. It also measures the degree of improvements since an earlier rating. The CAPS has demonstrated strong convergent validity with the Structured Clinical Interview for DSM-IV (SCID)-PTSD module $(r=.83)$ and the PTSD Symptom Scale Interview $(r=.73$; Foa $\&$ Tolin, 2000). The CAPS has also been found to have sound psychometric properties; test-retest reliabilities ranged from .77 to .96 for the three symptom clusters and from .90 to .98 for the 17 -item core symptom scale (Blake et al., 1995). The measure was administered five times: at baseline; immediately post-12week intervention; and 1,2, and 3 months post-treatment.

\section{Substance Use Measure}

Time Line Follow Back (TLFB). The TLFB is a clinician-assisted measure to assess recent drinking behavior over the 90-day period prior to the interview. Subjects retrospectively estimate their daily alcohol consumption while referencing a detailed calendar. The participant is oriented to specific episodes of regular, erratic, or binge drinking by the clinician. The TLFB is reliable and, in several studies, data obtained with a method like the TLFB have been sensitive to changes in alcohol consumption. The TLFB has high temporal stability with most test-retest correlations exceeding .85 (Sobell \& Sobell, 1995). The TLFB was used to assess alcohol and 
drug use patterns 90 days prior to treatment; it was then administered at each follow-up point (Sobell \& Sobell, 1992, 1995, 2000). The measure was administered five times: at baseline; immediately post-12-week intervention; and 1,2, and 3 months post-treatment.

\section{Emotion Regulation Measure}

Difficulties in Emotion Regulation Scale (DERS). The DERS is a 41-item self-report measure developed to assess clinically significant difficulties in emotion regulation. DERS items were chosen to reflect difficulties within the following dimensions of emotion regulation: (a) awareness and understanding of emotions; (b) acceptance and emotions; (c) the ability to engage in goal-directed behavior, and refrain from impulsive behavior when experiencing negative emotions; and (d) access to emotion regulation strategies perceived as effective. Responses range from 1 to 5, with higher scores indicating greater difficulties in emotion regulation. The total DERS was found to have good test-retest reliability $(r=.88)$ and high internal consistency $(\alpha=.93)$; the subscales also had high internal consistency (i.e., Cronbach's $\alpha$

$>.80$ for each). Preliminary findings suggest adequate construct and predictive validity (Gratz \& Roemer, 2004; Sloan \& Kring, 2007). The measure was administered five times: at baseline; immediately post-12-week intervention; and 1,2, and 3 months post-treatment.

\section{Physiological Arousal Measure}

Electrodermal skin fluctuation. Electrodermal skin fluctuation is an instrument commonly used in psychophysiology experiments to infer emotional state and cortical arousal (Ressler et al., 2004; Storm et al., 2002). This methodology measures electrical conductance in the skin through detecting variance in sweat gland activity. Because sweat glands become more active in association with the arousal, a higher number and amplitude of conductance fluctuations is thus correlated with heightened levels of stress (Storm, 2008). Using this 
physiological methodology to measure regulation of emotion strengthens the validity of selfreport emotion regulation findings.

The study utilized the ProComp2 electrodermal skin fluctuation device (produced by Thought Technology). Two sensors were placed on the participant's hand, one touching the palm and the second attached to the pointer finger. Once sensors were attached, a very slight electrical current ran through the electrodermal skin fluctuation device, which recognized variances in salt and water from sweat glands. When the skin's surface produces more sweat, the electrical conductivity increases, reflecting a higher reading on the device and indicating higher levels of stress. The electrodermal skin fluctuation measure was administered during each of the 12 individual therapy sessions.

Physiological arousal was measured weekly during sessions using skin conductance fluctuations per minute and two measures were explored. An average measurement was calculated across each session and change in average measurement was calculated. Secondly, each session was divided into 10-minute epochs. An average measurement was calculated for each 10-minute epoch and the peak rate epoch was determined. Change in peak rate epoch across sessions was calculated.

\section{Data Analysis}

All data were entered into SPSS Version 21.0. Initial frequencies were examined for outliers and checked against raw data. Exploratory analyses were performed on the data to create descriptive statistics on the sample. Alpha coefficients were computed to examine each scale's reliability. The associations between emotion dysregulation and other independent variables, such as treatment condition and symptom severity at baseline, were examined through correlation analyses. Relationships among dependent variables and demographic and 
background variables were performed using correlations, chi squares, $t$ tests, and ANOVAs to check for possible covariates. Research questions were answered using $2 \times 2 \times 2$ ANOVAs. See Figure 1 for sample $2 \times 2 \times 2$ ANOVA.

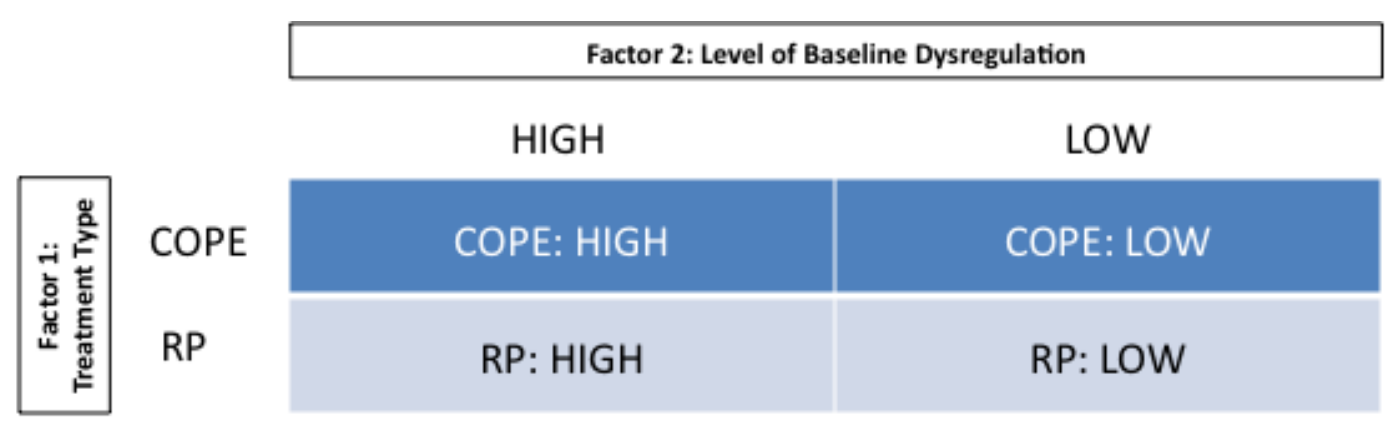

Figure 1. Will treatment type modify the relationship between baseline dysregulation, and outcomes of interest? 


\section{CHAPTER 4: RESULTS}

\section{Baseline Characteristics}

Table 1 depicts demographic characteristics in addition to variables related to pretreatment substance use, PTSD symptoms, and emotion dysregulation. To review, participants ranged in age from 20 to 64 , with a mean age of 39.8 years $(S D=11.9)$. The sample was $66.3 \%$ male $(n=53)$ and $33.8 \%$ female $(n=27)$. Over $75 \%$ of the participants indicated they were a racial/ethnic minority, with 56.3\% identified as African American $(n=45)$ and $21.3 \%$ identified as Hispanic $(n=17)$. Over $90 \%$ of the sample was born in the United States $(n$ $=73)$ and $93.5 \%$ of participants $(n=72)$ indicated that English was their first language. In terms of education, participants attended, on average, 13.07 years of schooling $(S D=3.0)$. At baseline assessment, $67.5 \%$ were employed in some fashion. Regarding service utilization prior to study enrollment, $23.6 \%$ of participants $(n=17)$ reported receiving previous outpatient treatment for psychological problems; 55.6\% $(n=40)$ reported receiving previous inpatient treatment for psychological problems; and $23.9 \%$ of participants $(n=17)$ reported previous trauma specific treatment. At baseline assessment, $54.1 \%$ of the sample $(n=40)$ indicated they had at some point been prescribed psychotropic medication and nearly $20 \%$ of the sample $(n=13)$ reported at least one suicide attempt.

Approximately $60 \%$ of participants met DSM-IV-TR criteria for PTSD $(n=50)$ while the remaining $37.5 \%$ demonstrated symptoms consistent with subthreshold PTSD $(n=30)$. The total sample had a mean DERS score of $90.9(S D=22.4)$. The subjects with DERS scores above the mean were considered to be high dysregulators $(48.8 \% ; n=39)$; participants with DERS scores below the mean were considered to be low dysregulators $(51.3 \% ; n=41)$. 
Table 1

Baseline Participant Characteristics

\begin{tabular}{|c|c|c|c|c|c|c|}
\hline \multirow[b]{2}{*}{ Variable } & \multicolumn{2}{|c|}{$\begin{array}{c}\text { Total } \\
(N=80)\end{array}$} & \multicolumn{2}{|c|}{$\begin{array}{l}\text { COPE } \\
(n=40)\end{array}$} & \multicolumn{2}{|c|}{$\begin{array}{c}\mathrm{RPT} \\
(n=40)\end{array}$} \\
\hline & $M$ or $\%$ & $S D$ & $M$ or $\%$ & $S D$ & $M$ or $\%$ & $S D$ \\
\hline Age & 39.8 & 11.9 & 38.8 & 11.9 & 40.8 & 12.0 \\
\hline Gender ( $\%$ female $)$ & 33.8 & & 35.0 & & 32.5 & \\
\hline \multicolumn{7}{|l|}{ Race/ethnicity (\%) } \\
\hline African American/Black & 56.3 & & 52.5 & & 60.0 & \\
\hline Caucasian & 18.0 & & 20.0 & & 17.5 & \\
\hline Latino & 21.3 & & 22.5 & & 20.0 & \\
\hline Other & 3.8 & & 5.0 & & 2.5 & \\
\hline \multicolumn{7}{|l|}{ Marital status (\%) } \\
\hline Married & 17.7 & & 15.0 & & 20.5 & \\
\hline Single & 25.3 & & 65.0 & & 48.7 & \\
\hline Divorced/separated & 57.0 & & 20.0 & & 30.8 & \\
\hline Years of education & 13.1 & 3.0 & 13.2 & 3.1 & 12.9 & 3.0 \\
\hline \multicolumn{7}{|l|}{ Employment (\%) } \\
\hline Employed & 67.5 & & 71.8 & & 63.2 & \\
\hline Unemployed & 14.3 & & 10.3 & & 18.4 & \\
\hline Student/retired/disabled & 18.2 & & 17.9 & & 18.4 & \\
\hline PTSD diagnosis (\% full) & 62.5 & & 65.0 & & 60.0 & \\
\hline CAPS total, Baseline ${ }^{\mathrm{a}}$ & 53.7 & 17.4 & 52.9 & 16.3 & 54.5 & 18.6 \\
\hline Substance use days in last 7 & 4.4 & 2.5 & 4.5 & 2.5 & 4.3 & 2.4 \\
\hline DERS total score, Baseline & 90.9 & 22.4 & 89.5 & 23.0 & 92.2 & 21.9 \\
\hline Therapy sessions attended & 6.7 & 4.6 & 5.8 & 4.6 & 7.5 & 4.5 \\
\hline \multicolumn{7}{|l|}{ Prior mental health treatment } \\
\hline Inpatient (\% Yes) & 55.6 & & 56.8 & & 54.3 & \\
\hline Outpatient (\% Yes) & 23.6 & & 22.9 & & 24.3 & \\
\hline $\begin{array}{l}\text { Trauma specific treatment } \\
\text { (\% Yes) }\end{array}$ & 23.9 & & 22.9 & & 25.0 & \\
\hline Treatment group (\% RPT) & 50.0 & & 0.0 & & 100.0 & \\
\hline
\end{tabular}

Note. Values are either means (with standard deviations) or percentages. COPE $=$ Concurrent Treatment of PTSD and Substance Use Disorders Using Prolonged Exposure; RPT = relapse prevention therapy. PTSD = posttraumatic stress disorder; CAPS = Clinician-Administered PTSD Scale; DERS = Difficulties in Emotion Regulation Scale. There were no statistical differences between treatment groups on any pretreatment variable.

${ }^{\text {a }}$ Variable included in randomization stratification. 
Half of the sample was randomized to the COPE treatment $(n=40)$ and half was randomized to RPT $(n=40)$. Overall, treatment attendance ranged from 0 to 12 sessions; participants attended a mean of 6.7 sessions $(S D=4.6)$. There were no statistically significant differences between treatment groups for the various baseline characteristics.

\section{Descriptive Statistics}

Table 2 depicts descriptive statistics for variables to be analyzed during correlation and regression analysis. CAPS, Substance use days in last 7, and DERS, variables were all within acceptable limits for skewness and kurtosis. All measures had good to excellent reliability $(\alpha>8)$.

In Table 3, correlation analyses examined potential non-hypothesized relationships between the dependent and independent variables. Pearson coefficients showed there was a significant positive association between the CAPS follow-up and days used follow-up, $r(41)=$ $.36, p=.02$; DERS baseline and DERS follow-up, $r(38)=.67, \mathrm{p}<.01$; CAPS baseline and CAPS follow-up, $r(41)=.57, \mathrm{p}<.01$; and CAPS baseline and DERS follow-up, $r(38)=.40, \mathrm{p}=$ .01 . These correlations, while not hypothesized, are in the expected direction.

\section{Research Question 1: Analysis}

Pearson correlation analyses were conducted to determine whether there were relationships among baseline emotion dysregulation, as measured by the DERS, and baseline PTSD and SUD, as measured by the CAPS score, and substance use days in the last 7 at baseline among individuals with PTSD. 
Table 2

Descriptive Statistics: Substance Use, Emotion Dysregulation, and PTSD Symptoms at Baseline

\begin{tabular}{|c|c|c|c|c|c|c|c|}
\hline Variable & $M$ & $S D$ & Min & Max & Skewness & Kurtosis & Cronbach's $\alpha$ \\
\hline CAPS baseline $(n=80)$ & 53.73 & 17.36 & 18.00 & 94.00 & .37 & -0.42 & .81 \\
\hline Substance use days in last 7 & 4.40 & 2.45 & 0.00 & 7.00 & -.39 & -1.21 & \\
\hline \multicolumn{8}{|l|}{ Baseline $(n=80)$} \\
\hline CAPS follow-up $(n=41)$ & 37.25 & 24.77 & 0.00 & 100.00 & .53 & -.37 & .93 \\
\hline Substance use days in last 7 & 1.83 & 2.41 & 0.00 & 7.00 & 1.05 & -.23 & \\
\hline \multicolumn{8}{|l|}{ Follow-up $(n=41)$} \\
\hline DERS follow-up $(n=38)$ & 80.22 & 21.18 & 46.00 & 124.00 & .25 & -1.05 & .93 \\
\hline
\end{tabular}




\section{Table 3}

Correlation Analysis of Independent and Dependent Variables

\begin{tabular}{|c|c|c|c|c|c|c|}
\hline & 1 & 2 & 3 & 4 & 5 & 6 \\
\hline 1. Days used baseline & - & & & & & \\
\hline 2. Days used follow-up & .14 & - & & & & \\
\hline 3. DERS baseline & -.12 & -.03 & - & & & \\
\hline 4. DERS follow-up & -.29 & .08 & $.674^{* *}$ & - & & \\
\hline 5. CAPS baseline & .14 & .17 & $.41^{* *}$ & $.40^{*}$ & - & \\
\hline 6. CAPS follow-up & .06 & $.36^{*}$ & .07 & .31 & $.54^{* *}$ & - \\
\hline
\end{tabular}

Note. CAPS $=$ Clinician-Administered PTSD Scale; DERS $=$ Difficulties in Emotion Regulation Scale ${ }^{*} p \leq 0.05 .{ }^{* *} p \leq 0.01$. 
As shown in Table 4, there was no significant correlation between the DERS baseline total score and substance use days in the last 7 at baseline. A statically significant positive association was found between the CAPS baseline total score and the DERS baseline total score, $r(80)=.41, p<0.01$, with DERS baseline total score explaining $17.4 \%$ of the variability in CAPS baseline total score. Baseline emotion dysregulation is associated with baseline PTSD symptoms but not with baseline substance use.

\section{Research Question 2: Analysis}

A $2 \times 2 \times 2$ (DERS group by treatment type by time) repeated measures ANOVA was conducted to determine whether treatment type moderates the relationship between baseline dysregulation, as measured by the DERS, and treatment outcomes, as measured by the CAPS and substance use days in the last 7 at follow-up.

Table 4

Correlation Analysis Among Baseline DERS, Substance Use Days in the Last 7 Days, and CAPS Scores (N = 80)

\begin{tabular}{|c|c|c|c|}
\hline Variable & 1 & 2 & 3 \\
\hline 1. DERS, Baseline & - & & \\
\hline 2. CAPS, Baseline & $.41 * *$ & - & \\
\hline $\begin{array}{l}\text { 3. Substance use days in } \\
\text { the last } 7, \text { Baseline }\end{array}$ & -0.12 & .14 & - \\
\hline
\end{tabular}


Table 5 presents the results of the multivariate tests and shows a significant time effect, $F(1,37)=25.78, p<.01$, partial $\eta^{2}=.59$, and a trend for the hypothesized $2 \times 2 \times 2$ interaction, $F(1,37)=2.7, p=.08$, partial $\eta^{2}=.13$. Since the sample size is fairly small and the effect size of the interaction effect was medium (explaining $13 \%$ of the variability in outcome measures), univariate tests were conducted. Tables 6 and 7 present results from the univariate tests.

As shown in Table 6, time was found to be significant for days used, $F(1,37)=28.67, p$ $<.01$, partial $\eta^{2}=.44$. Additionally, there was a DERS group by treatment group interaction on PTSD outcomes, regardless of time, $F(1,37)=5.06, p=.03$, partial $\eta^{2}=.12$. As a result, $t$ tests were used to determine specifically which groups differed significantly.

As shown in Table 8, among high dysregulators, the RPT group had higher mean CAPS total score $(M=57.04, S E=5.37)$ as compared to the COPE group $(M=42.83, S E=6.41)$ whereas among low dysregulators, RPT group did not have a significantly different mean CAPS total score as compared to the COPE group $\left(M_{\text {difference }}=5.32, S E=8.36, p=.53\right)$, regardless of time. This difference indicates an unintentional lack of randomization on the PTSD severity scores. See Figure 2 for a graphical depiction of the group differences.

As shown in Table 7, time was found to be significant for days used, $F(1,37)=33.84$, $p<.01$, partial $\eta^{2}=.48$. Additionally, the Time $\times$ Treatment Group $\times$ DERS Group interaction was found to be significant for days used, $F(1,37)=5.281, p<.027$, partial $\eta^{2}=.125$. As a result, $t$ tests were used to determine specifically which groups differed significantly. 
Table 5

Multivariate Tests: Change in Outcomes Over Time by Baseline DERS Group by Treatment Group $(\mathrm{N}=38)$

\begin{tabular}{|c|c|c|c|c|c|c|c|}
\hline \multirow[b]{2}{*}{ Effect } & & \multicolumn{6}{|c|}{ Pillai's Trace } \\
\hline & & Value & $F$ & $\begin{array}{l}\text { Hypothesis } \\
d f\end{array}$ & $\begin{array}{c}\text { Error } \\
\quad d f\end{array}$ & $p$ & $\begin{array}{c}\text { partial } \\
\eta 2\end{array}$ \\
\hline \multirow{4}{*}{ Between subjects } & Intercept & .90 & 160.78 & 2 & 36 & $.01^{* *}$ & .90 \\
\hline & DERS group & .11 & 2.26 & 2 & 36 & .12 & .11 \\
\hline & Treatment group & .06 & 1.18 & 2 & 36 & .32 & .06 \\
\hline & DERS group $\times$ Treatment group & .12 & 2.46 & 2 & 36 & .10 & .12 \\
\hline \multirow{4}{*}{ Within subjects } & Time & .59 & 25.78 & 2 & 36 & $.01^{* *}$ & .59 \\
\hline & Time $\times$ DERS group & .08 & 1.52 & 2 & 36 & .23 & .08 \\
\hline & Time $\times$ Treatment group & .04 & .66 & 2 & 36 & .52 & .04 \\
\hline & Time $\times$ DERS group $\times$ Treatment group & .13 & 2.70 & 2 & 36 & .08 & .13 \\
\hline
\end{tabular}

Note. CAPS = Clinician-Administered PTSD Scale; DERS = Difficulties in Emotion Regulation

${ }^{*} p \leq 0.05 .{ }^{* *} p \leq 0.01$. 
Table 6

Univariate Tests for PTSD Symptoms by Time by Treatment Group by DERS Group (N=38)

\begin{tabular}{lrrrrrr}
\hline \multicolumn{1}{c}{ Effect } & $M S$ & \multicolumn{1}{c}{$d f$} & \multicolumn{1}{c}{$S S$} & \multicolumn{1}{c}{$F$} & \multicolumn{1}{c}{$p$} & partial $\eta 2$ \\
\hline \multicolumn{1}{c}{ Within subject effects } & & & & & & \\
Time & 6344.48 & 1 & 6344.48 & 28.67 & $.01^{* *}$ & .44 \\
Time $\times$ DERS group & 591.60 & 1 & 591.60 & 2.67 & .11 & .07 \\
Time $\times$ Treatment group & 0.02 & 1 & 0.02 & .00 & .99 & .00 \\
Time $\times$ DERS group $\times$ Treatment group & 187.97 & 1 & 187.97 & .85 & .36 & .02 \\
Error & 221.30 & 37 & 8188.04 & & & \\
$\quad$ Between subjects effects & & & & & & \\
DERS group & 936.19 & 1 & 936.19 & 1.63 & .21 & .04 \\
Treatment group & 72.01 & 1 & 72.01 & 0.13 & .73 & .003 \\
DERS group $\times$ Treatment group & 2911.62 & 1 & 2911.62 & 5.06 & $.03^{*}$ & .12 \\
Error & 575.79 & 37 & 21304.10 & & & \\
& & & & & & \\
\hline
\end{tabular}

Note. CAPS $=$ Clinician-Administered PTSD Scale. DERS = Difficulties in Emotion Regulation.

${ }^{*} p \leq 0.05 ;{ }^{* *} p \leq 0.01$ 
Table 7

Univariate Analysis for Substance Use Symptoms by Time by Treatment Group by DERS Group $(\mathrm{N}=38)$

\begin{tabular}{lrrrrrr}
\hline \multicolumn{1}{c}{ Effect } & \multicolumn{1}{c}{ MS } & $d f$ & \multicolumn{1}{c}{$S S$} & \multicolumn{1}{c}{$F$} & $p$ & partial $\eta 2$ \\
\hline \multicolumn{1}{c}{ Within subject effects } & & & & & & \\
Time & 161.60 & 1 & 161.60 & 33.84 & $0.01^{* *}$ & 0.48 \\
Time $\times$ DERS group & 0.62 & 1 & 0.62 & 0.13 & .72 & 0.003 \\
Time $\times$ Treatment group & 6.31 & 1 & 6.31 & 1.32 & .26 & 0.03 \\
Time $\times$ DERS group $\times$ Treatment group & 25.22 & 1 & 25.22 & 5.28 & $.03^{*}$ & 0.13 \\
Error & 221.30 & 37 & 8188.04 & & & \\
$\quad$ Between subjects effects & & & & & & \\
DERS group & 11.07 & 1 & 11.07 & 1.66 & .21 & 0.04 \\
Treatment group & 12.14 & 1 & 12.14 & 1.82 & .19 & 0.05 \\
DERS group $\times$ Treatment group & 3.43 & 1 & 3.43 & 0.51 & .48 & 0.01 \\
Error & 6.69 & 37 & 247.34 & & & \\
& & & & & &
\end{tabular}

Note. CAPS $=$ Clinician-Administered PTSD Scale; DERS = Difficulties in Emotion Regulation. ${ }^{*} p \leq 0.05 .{ }^{* *} p \leq 0.01$.

Table 8

t Tests for Baseline DERS Group by Treatment Group on CAPS Score, Regardless of Time

\begin{tabular}{lccccc}
\hline \multicolumn{1}{c}{ DERS Group } & \multicolumn{1}{c}{$\begin{array}{c}\text { Treatment } \\
\text { Group }\end{array}$} & Mean $(S D)$ & $\begin{array}{c}\text { Mean } \\
\text { Difference }\end{array}$ & $S E$ & $p$ \\
\hline $\begin{array}{l}\text { Low } \\
\text { dysregulation }\end{array}$ & RPT $(n=14)$ & $37.80(4.54)$ & 5.32 & 8.36 & .53 \\
& COPE $(n=10)$ & $48.15(5.37)$ & & & \\
$\begin{array}{l}\text { High } \\
\text { dysregulation }\end{array}$ & RPT $(n=10)$ & $57.04(5.37)$ & & & \\
& COPE $(n=7)$ & $42.83(6.41)$ & & & $.009^{* *}$ \\
\end{tabular}

Note. CAPS $=$ Clinician-Administered PTSD Scale; DERS = Difficulties in Emotion Regulation. ${ }^{*} p \leq 0.05$. ${ }^{* *} p \leq 0.01$. 


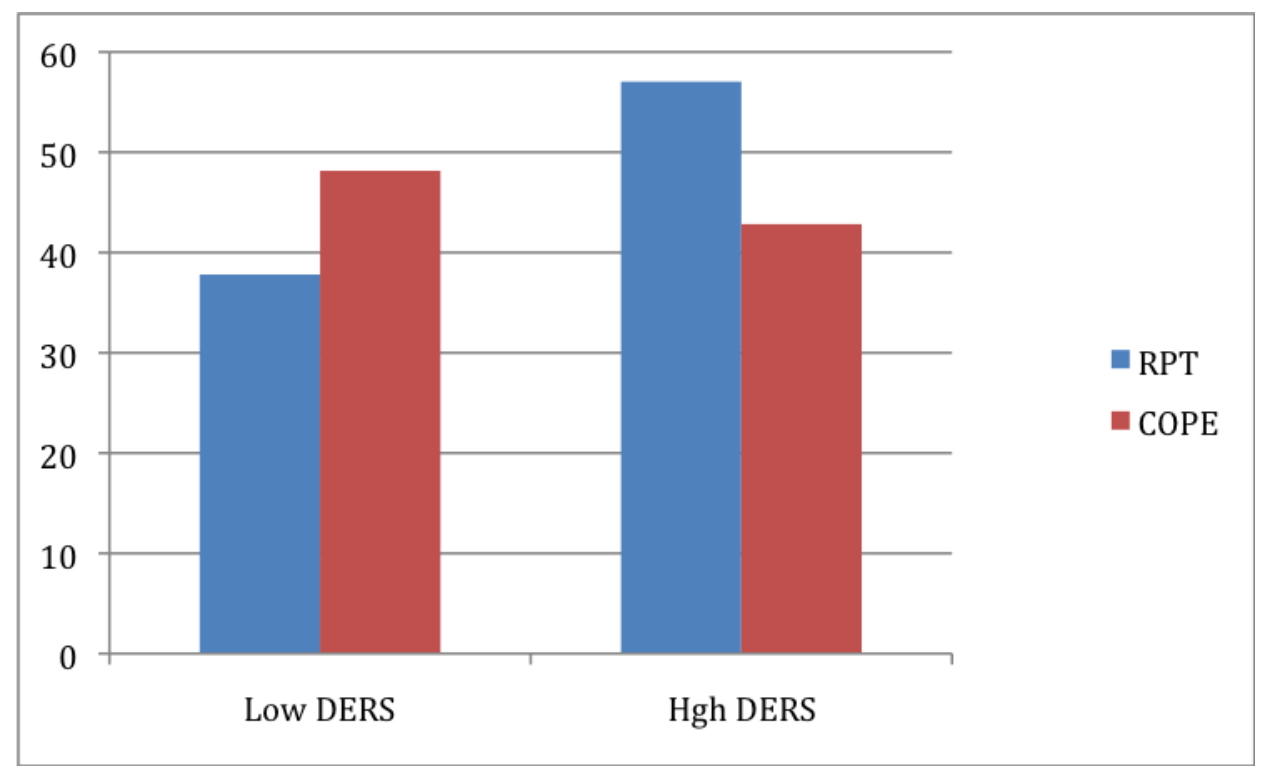

Figure 2. Baseline DERS group by treatment group on CAPS score $(N=41)$. CAPS = Clinician-Administered PTSD Scale; DERS = Difficulties in Emotion Regulation; COPE = Concurrent Treatment of PTSD and Substance Use Disorders Using Prolonged Exposure; RPT = relapse prevention therapy.

As shown in Table 9, the high baseline DERS group that received RPT treatment showed no change in days used. All other groups, that is the high baseline DERS group that received COPE treatment, the low baseline DERS group that received COPE treatment, and the low baseline DERS group that received RPT treatment, showed significant change in days used from baseline to follow-up (see Table 9 for means pre- and post-treatment). See Figure 3 for a graphical depiction of the interaction.

\section{Research Question 3: Analysis}

A $2 \times 2 \times 2$ (DERS group by treatment type by time) repeated measures ANOVA was conducted to determine whether treatment type moderates the relationship between baseline dysregulation, as measured by the DERS, and change in emotion dysregulation, as measured by change in DERS total score. 
Table 9

t Tests for Time by Treatment Group by DERS Group on Substance Use

\begin{tabular}{|c|c|c|c|c|c|c|}
\hline DERS Group & $\begin{array}{l}\text { Treatment } \\
\text { Group }\end{array}$ & Baseline $M(S D)$ & Follow-up $M(S D)$ & $t$ & $d f$ & $p$ \\
\hline Low dysregulation & $\begin{array}{l}\operatorname{RPT}(n=14) \\
\operatorname{COPE}(n=10)\end{array}$ & $\begin{array}{l}4.86(2.21) \\
5.50(2.46)\end{array}$ & $\begin{array}{l}1.21(2.33) \\
3.00(2.75)\end{array}$ & $\begin{array}{l}4.885 \\
2.277\end{array}$ & $\begin{array}{r}13 \\
9\end{array}$ & $\begin{array}{l}.001^{* *} \\
.049^{*}\end{array}$ \\
\hline $\begin{array}{l}\text { High } \\
\text { dysregulation }\end{array}$ & $\begin{array}{l}\operatorname{RPT}(n=10) \\
\operatorname{COPE}(n=7)\end{array}$ & $\begin{array}{l}3.20(2.94) \\
5.29(1.98)\end{array}$ & $\begin{array}{l}2.20(2.44) \\
0.86(1.46)\end{array}$ & $\begin{array}{l}0.866 \\
5.894\end{array}$ & $\begin{array}{l}9 \\
6\end{array}$ & $\begin{array}{l}.409 \\
.001^{* *}\end{array}$ \\
\hline
\end{tabular}

Note. CAPS = Clinician-Administered PTSD Scale; DERS = Difficulties in Emotion Regulation; COPE = Concurrent Treatment of PTSD and Substance Use Disorders Using Prolonged Exposure; RPT = relapse prevention therapy.

${ }^{*} p \leq 0.05 .{ }^{* *} p \leq 0.01$.

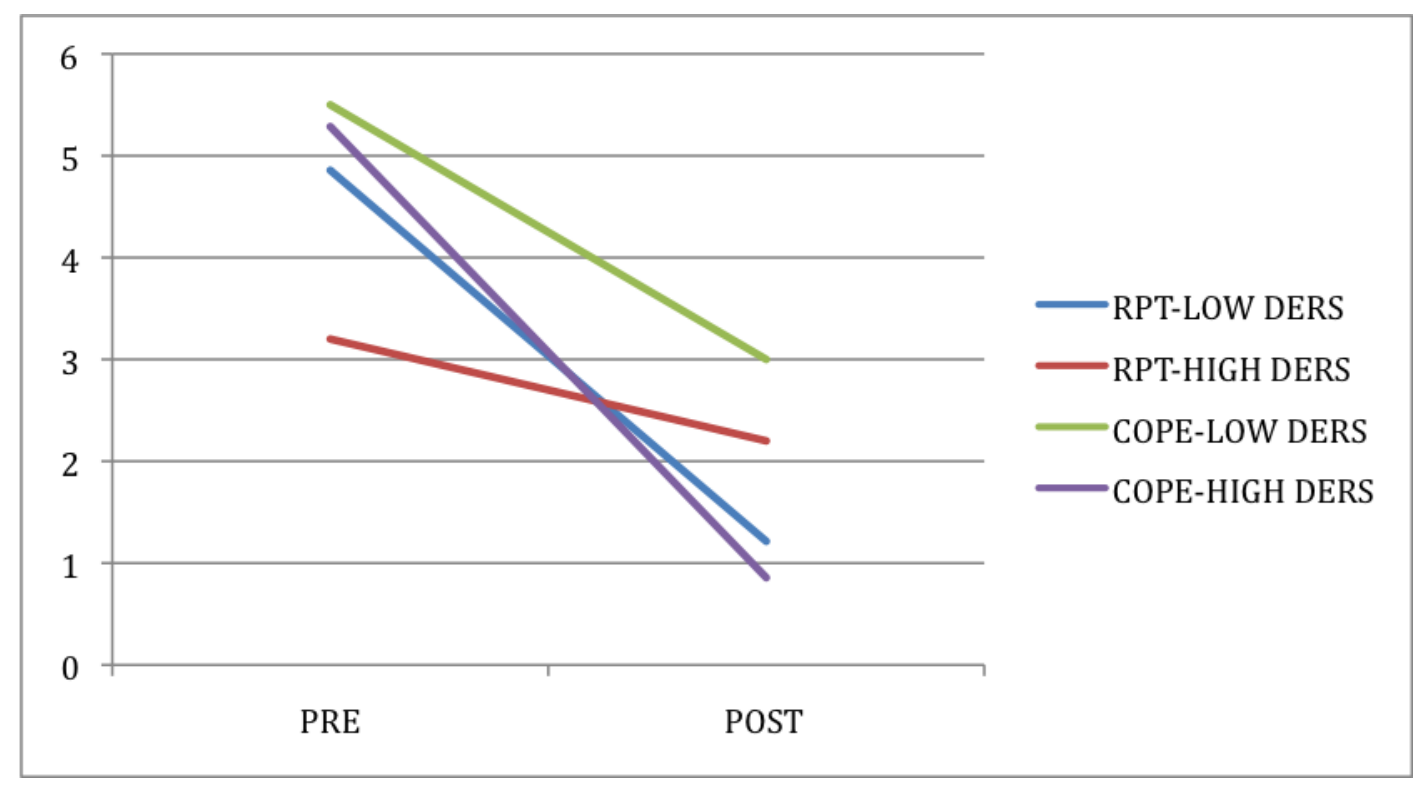

Figure 3. $t$ Tests for time by treatment group by DERS group on substance use $(n=41)$. COPE $=$ Concurrent Treatment of PTSD and Substance Use Disorders Using Prolonged Exposure; $\mathrm{RPT}=$ relapse prevention therapy. 
Table 10 presents the results of the univariate tests and shows a significant effect of time, $F(1,34)=7.11, p=.01$, partial $\eta^{2}=.17$, and a significant Time $\times$ DERS effect, $F(1,34)=6.25$, $p=.02$, partial $\eta^{2}=.16$, and a trend for the hypothesized $2 \times 2 \times 2$ interaction, $F(1,34)=3.40$, $p=.07$, partial $\eta^{2}=.09$ ). Since the sample size is fairly small and the effect size of the three-way interaction effect is small to medium (explaining $9 \%$ of the variability in outcome measures), post-hoc $t$ tests were conducted. Univariate tests also revealed a DERS group effect on change in DERS, regardless of time, $F(1,34)=45.96, p<.001$, partial $\eta^{2}=.58$. As a result, $t$ tests were used to determine specifically which groups differed significantly. Tables 11 through 13 present results from the post-hoc $t$ tests.

Table 10

Univariate Tests for Change in DERS by Time by Treatment Group by DERS Group ( $\mathrm{N}=38)$

\begin{tabular}{lrrrrrr}
\hline \multicolumn{1}{c}{ Effect } & \multicolumn{1}{c}{$M S$} & df & \multicolumn{1}{c}{$S S$} & \multicolumn{2}{c}{ partial } \\
\multicolumn{1}{c}{ Within subject effects } & & & & & \multicolumn{1}{c}{$\eta^{2}$} \\
Time & 908.05 & 1 & 908.05 & 7.11 & $.01^{* *}$ & .17 \\
Time $\times$ Treatment group & 24.62 & 1 & 24.62 & 0.19 & .66 & .01 \\
Time $\times$ DERS group & 798.61 & 1 & 798.61 & 6.25 & $.02^{*}$ & .16 \\
Time $\times$ DERS group $\times$ Treatment group & 434.06 & 1 & 434.06 & 3.40 & .07 & .09 \\
Error & 127.72 & 34 & 4342.32 & & & \\
$\quad$ Between subjects effects & & & & & & \\
Treatment group & 529.24 & 1 & 529.24 & 1.70 & .202 & .05 \\
DERS group & 14355.25 & 1 & 14355.25 & 45.96 & $.001^{* *}$ & .58 \\
DERS group $\times$ Treatment group & 704.02 & 1 & 704.02 & 2.25 & .142 & .06 \\
Error & 106118.74 & 34 & 106118.74 & & & \\
\end{tabular}

Note. DERS $=$ Difficulties in Emotion Regulation. ${ }^{*} p \leq 0.05 .{ }^{* *} p \leq 0.01$. 
Table 11

t Tests for Time by DERS Group on Change in DERS

\begin{tabular}{ccccccc}
\hline DERS Group & $n$ & $\begin{array}{c}\text { Baseline } \\
M(S D)\end{array}$ & $\begin{array}{c}\text { Follow-Up } \\
M(\mathrm{SD})\end{array}$ & $t$ & $d f$ & $p$ \\
\hline Low dysregulation & 23 & $71.72(10.44)$ & $70.78(16.06)$ & .29 & 22 & .77 \\
High dysregulation & 15 & $107.40(13.66)$ & $94.71(20.22)$ & 2.82 & 14 & $.01^{*}$
\end{tabular}

Note. DERS $=$ Difficulties in Emotion Regulation; COPE $=$ Concurrent Treatment of PTSD and Substance Use Disorders Using Prolonged Exposure; RPT = relapse prevention therapy.

${ }^{*} p \leq 0.05 .{ }^{* *} p \leq 0.01$.

Table 12

t Tests for Time by Treatment Group by DERS Group on Change in DERS

\begin{tabular}{cllrrrr}
\hline DERS Group & Treatment Group & Baseline $M(S D)$ & Follow-Up $M(S D)$ & $t$ & $d f$ & $p$ \\
\hline \multirow{2}{*}{ Low dysregulation } & RPT $(n=13)$ & $73.00(12.1)$ & $68.77(18.4)$ & 0.92 & 12 & .377 \\
& COPE $(n=10)$ & $70.07(8.12)$ & $73.39(12.85)$ & -0.78 & 9 & .458 \\
High dysregulation & RPT $(n=9)$ & $109.66(10.68)$ & $101.89(16.98)$ & 1.66 & 8 & .135 \\
& COPE $(n=6)$ & $104.00(17.8)$ & $83.92(21.25)$ & 2.38 & 5 & .063 \\
\hline
\end{tabular}

Note. DERS = Difficulties in Emotion Regulation; COPE = Concurrent Treatment of PTSD and Substance Use Disorders Using Prolonged Exposure; RPT = relapse prevention therapy.

${ }^{*} p \leq 0.05 ;{ }^{*} p \leq 0.01$

Table 13

t Tests for Baseline DERS group on Change in DERS, Regardless of Time

\begin{tabular}{rcccc}
\hline DERS Group $(n)$ & Mean $(S D)$ & Mean & \\
Difference & $S E$ & $p$ \\
\hline Low DERS group (24) & $71.31(2.63)$ & 28.56 & 4.21 & $0.01^{\text {*** }}$ \\
High DERS group (17) & $99.87(3.30)$ & & & \\
\hline
\end{tabular}

Note. DERS = Difficulties in Emotion Regulation Scale.

${ }^{*} p \leq 0.05$. ${ }^{*} p \leq 0.01$. 
As shown in Table 11, the high baseline DERS group showed a significant change in DERS at follow-up $\left(M_{\text {pre }}=107.40, S D=13.66 ; M_{\text {post }}=94.71, S D=20.22, p=.01\right)$ as compared to the change in DERS found in the low baseline DERS group at follow-up.

As shown in Table 12, the high baseline DERS group that received COPE treatment showed a change in DERS at follow-up at a trend level $\left(M_{\text {pre }}=104.00, S D=17.80 ; M_{\text {post }}=\right.$ 83.93, $S D=21.25, p=.063)$. All other groups, that is the high baseline DERS group that received RPT, the low baseline DERS group that received COPE treatment, and the low baseline DERS group that received RPT, showed no significant change DERS total from baseline to follow-up (see Table 12 for means pre- and post-treatment). See Figure 4 for a graphical depiction of the interaction.

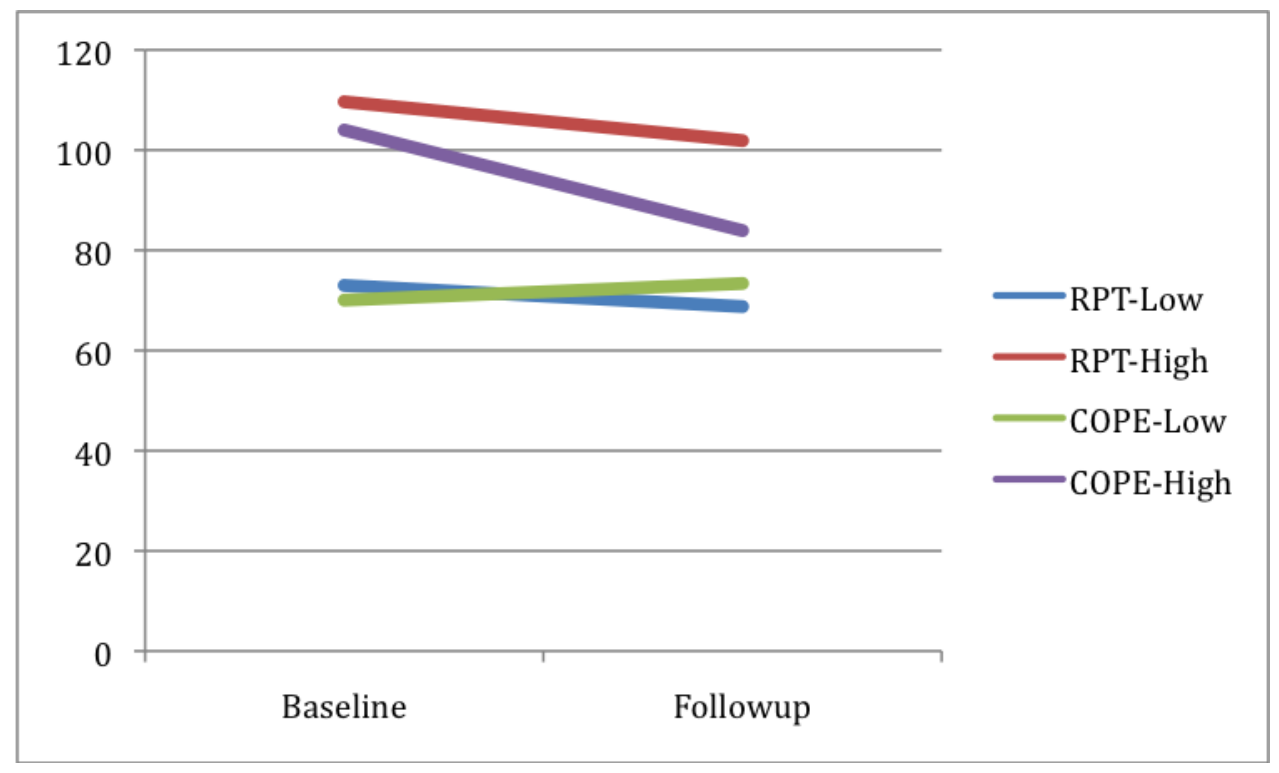

Figure 4. $t$ Tests for time by treatment group by DERS group on change in DERS $(n=38)$. 
Lastly, as shown in Table 13, the high DERS group had higher mean DERS total score $(M=99.87, S E=3.30)$ as compared to the low DERS group $(M=71.31, S E=2.63)$, regardless of time. This difference is a result of the high-low DERS grouping created for the $2 \times 2 \times 2$ analysis.

\section{Case Illustration}

The high baseline DERS group that received COPE treatment showed a trend level change in DERS at follow-up $\left(M_{\text {pre }}=104.00, S D=17.80 ; M_{\text {post }}=83.93, S D=21.25, p=.063\right)$. All other groups, specifically the high baseline DERS group that received RPT treatment, the low baseline DERS group that received COPE treatment, and the low baseline DERS group that received RPT treatment, showed no significant change DERS total from baseline to follow-up. To further explore the change in DERS over time among highly dysregulated individuals undergoing COPE, one case was selected for a case illustration. The participant's physiological arousal during sessions was examined using a skin conductance measure and both skin conductance level, a measure of overall arousal level, and skin conductance rate, a measure of the number of fluctuations in arousal per minute, were calculated.

Table 14 depicts demographic characteristics in addition to variables related to pretreatment and follow-up substance use, PTSD symptoms, emotion dysregulation, and physiological arousal. To review, the participant was a 51-year-old African American/Black male with some college education. He received 12 sessions of treatment and showed a 38-point reduction in emotion dysregulation, as measured by the DERS; a 28-point decrease in CAPS score; and a 6-day decrease in substance use days in the last 7 at follow-up. 
Table 14

Case Illustration Participant Characteristics

\begin{tabular}{lc}
\hline \multicolumn{1}{c}{ Variable } & $\begin{array}{c}\text { COPE } \\
(n=1)\end{array}$ \\
\hline Age & 51 \\
Gender (\% male) & 100 \\
Race/ethnicity (\%) & \\
$\quad$ African American/Black & 100 \\
$\quad$ Caucasian & 0 \\
$\quad$ Latino & 0 \\
$\quad$ Other & 0 \\
Years of education & 14 \\
Therapy sessions attended & 12 \\
Baseline DERS group (\% high dysregulation) & 100 \\
DERS total score, Baseline & 98 \\
DERS total score, Follow-up & 60 \\
CAPS total, Baseline & 28 \\
CAPS total, Follow-up & 0 \\
Substance use days in last 7, Baseline & 7 \\
Substance use days in last 7, Follow-up & 1 \\
SCR, Session 1 (peaks per minute) & 1.61 \\
SCR, Session 12 (peaks per minute) & 1.06 \\
\% Change in SCR & -34 \\
SCL, Session 1 & 2.41 \\
SCL, Session 12 & 1.34 \\
\% Change in SCL & -44 \\
& \\
\hline &
\end{tabular}

Note. Values are either raw scores or percentages. COPE $=$ Concurrent Treatment of PTSD and Substance Use Disorders Using Prolonged Exposure. PTSD = posttraumatic stress disorder; CAPS = Clinician-Administered PTSD Scale; DERS = Difficulties in Emotion Regulation Scale; $S C R=$ skin conductance rate; $S C L=$ skin conductance level.

Figure 5 depicts the physiological arousal over the course of treatment. With regards to physiological arousal, as measured by SCR, the COPE participant showed a steady increase in physiological arousal, peaking in the fourth session before his arousal decreased over the course of the last eight sessions. 


$$
3.5
$$

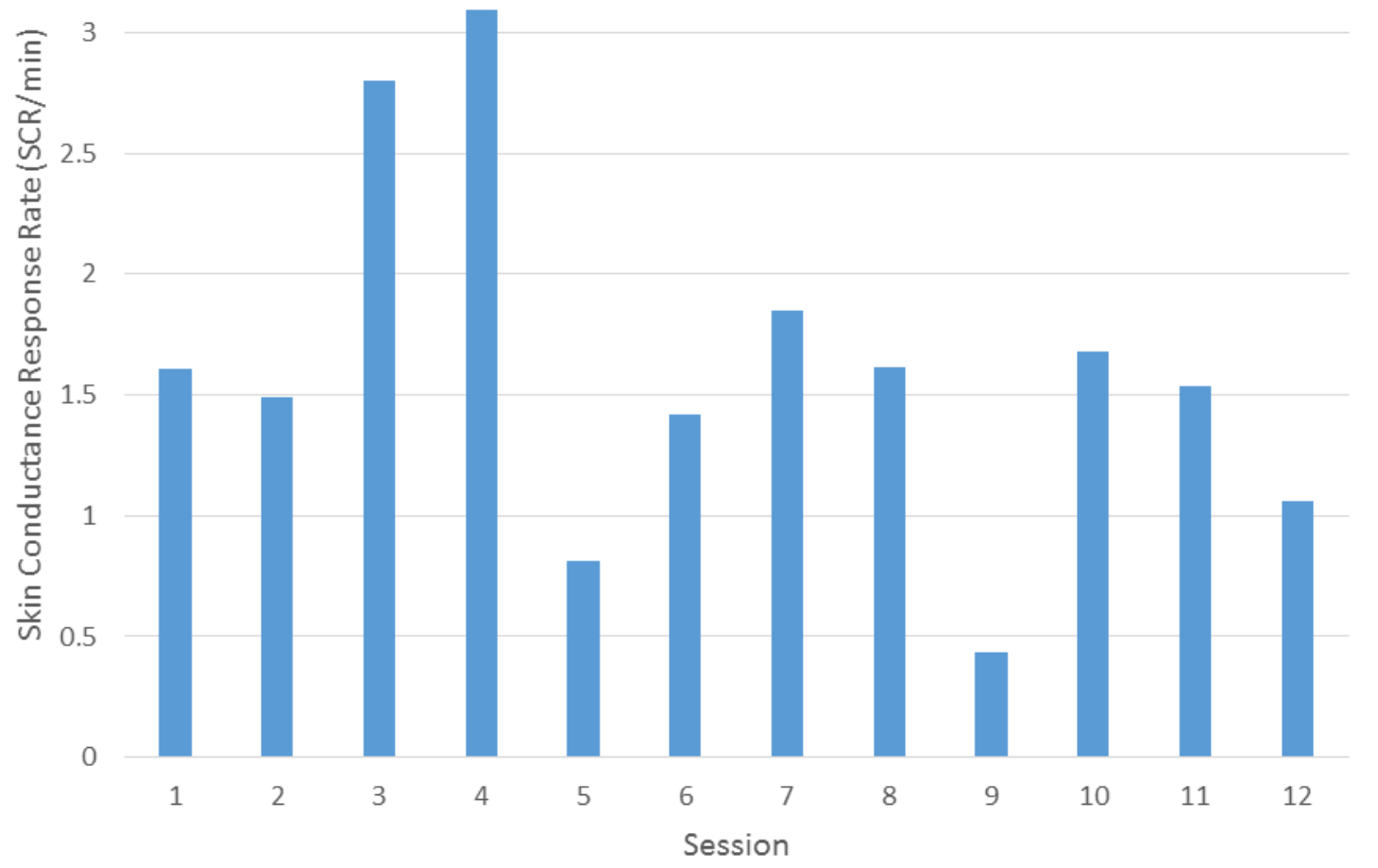

Figure 5. Change in physiological arousal through the course of treatment.

Figure 6 depicts the COPE participant's skin conductance during the first and last (12th) COPE session. The COPE participant had an average SCR of 1.61 peaks per minute and an average SCL of 2.41 micro Siemens during Session 1. During the 12th session, the COPE participant had an average SCR of 1.06 peaks per minute and an average SCL of 1.34 micro Siemens. As compared to Session 1, the COPE participant's overall arousal was 44\% lower with $34 \%$ fewer fluctuations during Session 12, indicating a lower level of arousal during the final session of treatment as compared to the initial session. 


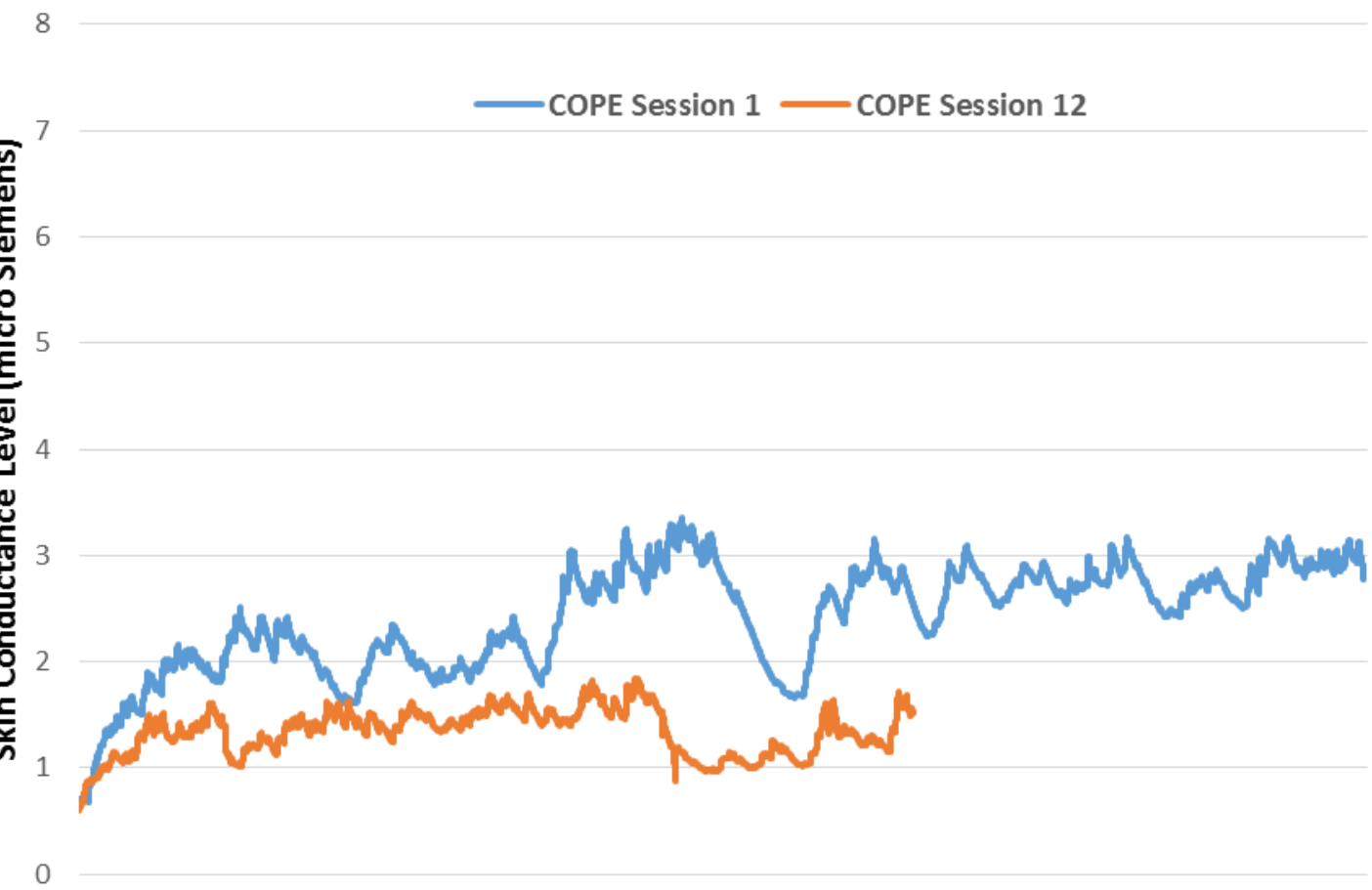

Minutes

Figure 6. Skin conductance during first and last COPE session. 


\section{CHAPTER 5: DISCUSSION}

Emotion dysregulation has been observed to be a shared feature of both PTSD and SUD (Hien et al., 2005). Treatments for these complex comorbid problems utilize a variety of approaches. However, to date, few studies have examined the effect of the various available treatments on emotion dysregulation in this population. The present study attempted to extend the research on emotion dysregulation among the comorbid PTSD and SUD population. It examined features associated with emotion dysregulation, associating dysregulation with other psychiatric symptoms affecting this clinical population. Specifically, the study focused on baseline emotion dysregulation as a predictor of treatment outcomes and used a longitudinal design to assess changes in emotion dysregulation over time to determine the mutability of this complex factor in response to treatment. Given the seriousness and prevalence of PTSD and SUD (van Dam et al., 2010), and the many impediments they pose to treatment efficacy, a more refined understanding of emotion dysregulation could support improved conceptualization and implementation of psychotherapeutic interventions for this population.

A detailed discussion of study findings is presented in the next five sections, beginning with an overview of the main findings, briefly summarizing the results related to each of the research questions. Additionally, research and clinical implications will be reviewed. Lastly, limitations and future directions for research will be presented.

\section{Summary of Main Findings}

Emotion dysregulation is inherent to many forms of psychopathology (Bradley et al., 2011). Previous studies using non-clinical and community samples found associations between PTSD symptom severity and overall emotion dysregulation (Weiss et al., 2013). Similar associations were observed among a treatment-seeking cocaine dependent sample (McDermott et 
al., 2009). People with severe PTSD and SUD symptoms are assumed to have high dysregulation. This study examined features associated with baseline emotion dysregulation among a comorbid PTSD and SUD population and indeed, found a positive association between the CAPS baseline total score and the DERS baseline total score. However, no significant correlation between the DERS baseline total score and substance use days in the last 7 at baseline was found. Baseline emotion dysregulation was associated with baseline PTSD symptoms, but not with baseline substance use among people with comorbid PTSD and SUD. The present study reinforces the findings of Weiss et al. (2013); Bonn-Miller, Vujanovic, Boden, and Gross (2011); and Tull, Barrett, McMillan, and Roemer (2007). Greater difficulty in regulating emotions is associated with PTSD symptom severity. Future research studies involving similar populations should take these findings into consideration.

Self-medicating with alcohol and drugs can provide temporary relief from the dysregulated feeling states that are characteristic of PTSD (Hien et al., 2009). Because of their long-standing chronic use of substances to manage affect, individuals with PTSD and SUD may not be aware of experiencing problems with emotion regulation. Unlike the CAPS which is a 30-item clinically administered interview protocol that is able to capture the nuances of multiple PTSD symptoms, the substance use measure utilized in this study focused on substance use frequency alone. It is noteworthy that frequency is only one symptom of a SUD and may likely not have been a sensitive enough measure to capture how emotion regulation and substance use are related. Additionally, though this study considered several aspects of emotion dysregulation including, impulse control, emotional awareness, access to regulation strategies, and emotional clarity, it is possible that the construct did not tap into addiction-related symptoms. It is also possible that certain episodic substance use patterns are not correlated with the DERS, as the 
measure asks for an overall rating of one's ability to manage strong emotions. Episodic use may be indicative of varying levels of emotion dysregulation. In sum, it is possible that use needs to be at a certain level and/or with a certain frequency to be correlated with dysregulation.

Exposure-focused treatments and skills-based treatments are premised on different emphases with respect to emotion dysregulation. RPT aims to change behavior through a topdown approach, examining cognitions in relation to affect. COPE, the only exposure treatment developed to treat SUD, utilizes a bottom-up fear habituation processing model. Specifically, the second research question in the present study explored whether treatment type moderated the relationship between baseline dysregulation, as measured by the DERS, and treatment outcomes, as measured by the CAPS and substance use days in the last 7 at follow-up. Findings revealed a significant three-way interaction: Time $\times$ Treatment Group $\times$ DERS Group was significantly different across the substance use outcome measure. Specifically, the high baseline DERS group that received COPE treatment, the low baseline DERS group that received COPE treatment, and the low baseline DERS group that received RPT treatment all showed significant change in number of days of use from baseline to follow-up; by contrast, the high baseline DERS group that received RPT treatment showed no change in number of days of use.

These findings suggest that among highly dysregulated individuals with SUD and PTSD, a treatment focusing solely on the SUD will not garner positive changes in substance use frequency. These results provide a strong indicator that treatments that do not directly discuss or integrate trauma cannot shift problematic substance use, especially among the highly dysregulated participants. Not only is a treatment like COPE indicated, but indeed these findings provide some suggestion that RPT may be contraindicated or even iatrogenic for highly dysregulated people with comorbid PTSD and SUD. These findings support Brady et al. (2001), 
Najavits et al. (2005), and most recently, Mills et al. (2012), in challenging the commonly held view that patients with comorbid PTSD and SUD are inappropriate candidates for prolonged exposure (Becker, Zayfert, \& Anderson, 2004). Though it has been theorized that individuals with comorbid PTSD and SUD are too high risk to engage in exposure-based treatments, empirical research including the present study, have shown evidence to the contrary. Patients with comorbid PTSD and SUD have shown the ability to tolerate and benefit from traumafocused treatment.

Additionally, treatment group by DERS group, regardless of time, was found to be significant for PTSD symptoms indicating a difference in the level of PTSD symptoms between the two treatment groups. More specifically, among high dysregulators, the RPT group had higher mean CAPS total score as compared to the COPE group, whereas among low dysregulators, RPT group did not have a significantly different mean CAPS total score as compared to the COPE group, regardless of time. This difference indicates an unintentional lack of randomization at baseline. Specifically, since participants were not matched on baseline emotion dysregulation during the randomization phase of the study, the RPT group had higher CAPS total score, regardless of time. The groups were not equivalent on this variable.

Randomization presumes equivalence across groups. If equivalence is not established, group comparisons are problematic. It is difficult, however, to always know which variables are important and should be considered in the randomization process. Since the present study evidenced that emotion dysregulation is related to PTSD symptom severity, future studies focusing on individuals with PTSD should consider baseline dysregulation as a factor for randomization. One challenge to considering baseline dysregulation in the randomization process is the difficulty in defining high verses low dysregulation in the study sample during the 
randomization phase. The present study calculated a sample mean DERS score and grouped those above the mean into the high dysregulation group and those below the mean into the low dysregulation group. Randomizing on dysregulation would require the a priori classification of dysregulation severity, which is not yet established in the literature.

Emotion regulation describes the processes by which individuals influence expression of emotions, when emotions arise, and how they are experienced (Hien et al., 2009). To the extent that certain emotion regulatory strategies are theorized to be more adaptive than others, it can be hypothesized that psychologically based treatments may affect emotion regulatory capacities. For example, individuals with comorbid PTSD and SUD often have a limited range of accessible emotional coping skills and are frequently over reliant on avoidant strategies in an attempt to manage intense feelings. Exposure therapy, directly and experientially, addresses emotion regulation problems and associated physiological arousal through habituation to disrupted fear responses in the service of assimilating and integrating overwhelming experiences. RPT for comorbid PTSD and SUD, on the other hand, focuses on identifying emotional states for the purpose of coping and managing intense emotion, so the treatment mainly works on developing skills and building coping strategies.

Specifically, the third research question in the present study examined whether treatment type moderated the relationship between baseline dysregulation and dysregulation at follow-up. Findings revealed (at a trend level) that those in the high baseline DERS group who received COPE treatment had lower DERS at follow-up. In contrast, all other groups, specifically the high baseline DERS group that received RPT, the low baseline DERS group that received COPE treatment, and the low baseline DERS group that received RPT, showed no significant change DERS total from baseline to follow-up. 
These results provide a speculation that among people with PTSD and SUD, those with high levels of emotion dysregulation may be particularly able to make use of the COPE treatment model. Engagement in a treatment that directly addresses trauma memories may positively impact emotion regulation capacities among those with particular difficulty in that domain. By reducing anxiety and increasing awareness, individuals no longer need to engage in emotional and behavioral avoidance. By confronting the trauma directly, it is possible to find some degree of emotional mastery over overwhelming traumatic experiences.

Additionally, the supplemental analysis of the skin conductance data provided descriptive information that depicted the physiological arousal of one participant in the high baseline DERS group who received COPE treatment. The participant was a 51-year-old African American/Black male with some college education. He received 12 sessions of treatment and showed a 38-point reduction in emotion dysregulation as measured by the DERS; a 28-point decrease in CAPS score; and a 6-day decrease in substance use days in the last 7 at follow-up. His physiological arousal pattern showed a decrease in arousal over time and was consistent with the change (at a trend level of $p=.063$ ) found in the high dysregulation COPE group's change in DERS at follow-up.

\section{Clinical Implications}

Experts in the field of substance abuse treatment have historically been hesitant to use prolonged exposure to treat individuals with comorbid PTSD and SUD. Exposure-based behavioral therapies are suspected to be contraindicated for people with addictions for fear of relapse, treatment dropout, increased acting-out behaviors, and dysregulation (van Minnen et al., 2012). In sum, prolonged exposure has been considered inappropriate for use among patients 
with SUD as it was believed that these patients may be unable to cope with the intense emotions triggered during exposure to trauma memories (Foa \& Rothbaum, 1998).

According to the results of this dissertation study, highly dysregulated persons with PTSD and SUD undergoing COPE exhibited positive changes in emotion dysregulation, while the other groups did not. Additionally, highly dysregulated individuals at baseline benefitted more from COPE than RPT with respect to change in substance use. While RPT is an intellectual skill-based approach focusing on cognitions and behavior, COPE uses both cognitive strategies and emotion-focused exercises to more experientially and directly address affective experiences. These results provide additional support for the use of integrated treatments for comorbid SUD and PTSD (Back, Waldrop, Hien, \& Brady, 2006; Hien, Campbell et al., 2010) among highly dysregulated individuals.

The sequential model of care for comorbid PTSD and SUD treats the substance use first and defers treatment of trauma-related issues. The clinical rationale behind deferring trauma treatment relates to a fear of that unaddressed substance abuse could impede other therapeutic efforts and potentially cause relapse (Pitman et al., 1991; as cited in Back et al., 2006). While RPT is often considered the gold standard addiction treatment (Carroll et al., 1994; as cited in Hien et al., 2004) the results from this exploratory study indicate that high baseline dysregulation renders RPT ineffective in a comorbid PTSD and SUD population. This exploratory study highlights the importance of directly and explicitly addressing trauma to improve substance use outcomes for highly dysregulated individuals with this comorbidity.

Patients sharing DSM-IV-TR diagnoses of PTSD and SUD are not a homogenous population. This analysis demonstrated that a one-size-fits-all approach has limited value for such comorbid conditions and underscored the importance of assigning patients to treatments 
based on their specific needs and characteristics. Specifically, different degrees of emotion dysregulation should be accounted for in order to provide a patient with matched clinical care. This dissertation indicates that an assessment of pretreatment emotion dysregulation can assist treatment efficacy. Additionally, this exploratory study furthers the work of Mills et al. (2012) by emphasizing that highly dysregulated populations with PTSD and SUD are able to both tolerate and benefit from exposure treatment.

Traditional intervention research has focused on comparing how groups of individuals receiving an experimental treatment compared to a control group. This approach provides information about treatments for selected groups of people, but says little about choosing the best treatment for a specific individual. As the health care landscape shifts to providing more personalized care, it is important to consider individual characteristics, like emotion dysregulation, to optimize outcomes. Researchers are developing ways to better predict patients' responses to targeted treatments; however, determining which factors have the most clinical significance is paramount (Hamburg \& Collins, 2010). The success of personalized medicine depends on having accurate assessments that identify patients who can benefit from targeted therapies (Hamburg \& Collins, 2010). This study may help to guide treatment customization based on interactions between emotion dysregulation and intervention focus. Trauma processing psychotherapies, like COPE, that provide opportunity for meaning-making and trauma integration, may be better aligned for individuals who struggle in those domains as they pertain to impairments in emotion dysregulation. 


\section{Research Implications}

Findings from an ANOVA identified a relationship between pretreatment levels of dysregulation and CAPS scores by treatment type. Among high dysregulators, the RPT group had higher mean CAPS total score as compared to the COPE group, whereas among low dysregulators, the RPT group did not have a significantly different mean CAPS total score as compared to the COPE group, regardless of time. This difference is indicative of an unintentional lack of randomization at baseline. This finding suggests that it is appropriate to stratify study samples by emotion dysregulation, as it is frequently associated with outcomes of interest in a comorbid PTSD and SUD population. Future studies involving samples with PTSD should consider randomizing on this factor.

\section{Limitations and Future Directions}

This exploratory study suggests the importance of directly discussing trauma in treatment for highly dysregulated people with comorbid PTSD and SUD. These results, however, must be interpreted with caution due to the small sample size. The interaction effects demonstrated within this dissertation indicate the importance of continuing the essential line of inquiry into emotion dysregulation and treatment matching with studies using larger samples.

Emotion dysregulation is known to be correlated with substance use severity (Suh et al., 2008); however, this study demonstrated that baseline emotion dysregulation was not associated with baseline substance use frequency among people with comorbid PTSD and SUD. Future research on this topic should look deeper into the data to address a more complete picture of the relationship between baseline substance use and baseline emotion dysregulation. Future studies should include measures of not only substance use frequency, but of quantity, cravings, and periods of abstinence. The Addiction Severity Index composite score, for example, could be 
used to better capture the complexities of substance use severity, as it accounts for the impact of substance use on living. Additionally, this study collapsed all categories of substances together (i.e., alcohol, cannabis, cocaine, etc.) when describing substance use. Alternatively, separating out classes of drugs may provide a more accurate picture of use patterns.

Emotion dysregulation has multiple facets. This study used a total composite score from the DERS self-report measure. Future research might benefit from looking at specific dimensions of emotion dysregulation. For example, the DERS can be broken down into four indices: (1) awareness and understanding of emotions; (2) acceptance of emotions; (3) the ability to engage in goal-directed behavior, and refrain from impulsive behavior when experiencing negative emotions; and (4) access to emotion regulation strategies perceived as effective. These indices may all function differently with respect to PTSD and SUD. Additionally, future studies may benefit from using a less dichotomous measure of emotion dysregulation. By acknowledging that emotion dysregulation exists on a spectrum, future studies might be advantaged by considering certain levels of emotional expressiveness or dysregulation to be advantageous, normative, or even healthy.

This study relied heavily on the use of an emotion dysregulation self-report measure. The case illustration, however, utilized skin conductance data. The use of physiological measures on a larger scale would address both the less subjective and less conscious aspects of arousal and potentially dysregulation. While skin conductance is widely used to index sympathetic arousal, it generally does not differentiate between positive and negative emotion (Dawson, Schell, \& Filion, 2007). Additionally, approximately 10 to $25 \%$ of participants are estimated to be hypo-responsive with respect to their electrodermal response; it may not be possible to obtain high quality skin conductance data measurements from some individuals 
(Braithwaite, Watson, Jones, \& Rowe, 2013). Lastly, external factors such as temperature and internal factors such as medication can affect galvanic skin response measurements, which can lead to inconsistent results. Future studies may include heart rate variability as an additional or alternative physiological measure of arousal. Research has shown that heart rate variability reflects sympathetic as well as parasympathetic activation, and it is sensitive to changes in arousal (Thayer, Ahs, Fredrickson, Stollers, \& Wager, 2012).

Lastly, functional neuroimaging studies have implicated multiple regions of the brain, including the right ventrolateral prefrontal cortex, amygdala, and dorsal anterior cingulate cortex, in the regulation of positive and negative emotions (Etkin, Egner, \& Kalisch, 2011; Ochsner \& Gross, 2005; Kalisch, 2009; Kanske, Heissler, Schönfelder, \& Wessa, 2011). It has been observed that dysfunction in these regions can lead to disturbances in emotional regulation (i.e., hypo- or hyper-arousal) and contribute significantly to impaired functioning underlying a variety of psychological disorders, including PTSD and SUD (Teicher, 2000; Cooper et al., 1995; Thorberg, \& Lyvers, 2006). Further, fMRI studies directly examining the neural correlates of emotion regulation among clinical populations could help to clarify the basic mechanisms by which emotions are regulated and demonstrate the efficacy of specific treatments to ameliorate deficits in this domain.

\section{Conclusion}

As a contribution to research and clinical practice, the current exploratory project provided new evidence that exposure therapy can be tolerated by and beneficial to highly dysregulated persons with PTSD and SUD. Contrary to expectations in the field, participants randomized to receive the exposure-based intervention did not demonstrate poorer substance use outcomes relative to those randomized to receive RPT. In fact, the present study findings 
provide strong support for the COPE treatment as more efficacious than RPT in reducing emotion dysregulation and substance use frequency among individuals with high levels of baseline dysregulation. Furthermore, this dissertation suggests that psychotherapy focusing only on substance use (e.g., RPT) may be contraindicated for highly dysregulated individuals with comorbid PTSD and SUD, as their substance use frequency did not decrease over time. This study supports the idea that the complex presentations of individuals with PTSD and SUD should not be a deterrent to providing trauma-focused treatment.

Knowing that specific alignments of emotion dysregulation and treatment types have differential effects on therapeutic outcomes helps to better match individuals with treatments and provide ways for therapy to be more effective. This exploratory study adds to what is known about patient characteristics as pretreatment predictors of outcome and enhances the potential of pairing patients with the types of treatment most likely to be helpful. 


\section{REFERENCES}

Aharonovich E., Nguyen H. T., \& Nunes, E. V. (2001). Anger and depressive states among treatment- seeking drug abusers: Testing the psychopharmacological specificity hypothesis. American Journal of Addictions, 10, 327-334. doi:10.1080/aja.10.4.327.334

Allen, J. G., \& Fonagy, P. (2006). Handbook of mentalization-based treatment. Chichester, United Kingdom: Wiley.

Aldao, A., Nolen-Hoeksema, S., \& Schweizer, S. (2010). Emotion-regulation strategies across psychopathology: A meta-analytic review. Clinical Psychology Review, 30, 217-237. doi:10.1016/j.cpr.2009.11.004

American Psychiatric Association. (2000). Diagnostic and statistical manual of mental disorders (4th ed., text rev.). Washington, DC: Author.

American Psychiatric Association. (2013). Diagnostic and statistical manual of mental disorders (5th ed.). Arlington, VA: American Psychiatric Publishing.

Back, S. E. (2010). Toward an improved model of treating co-occurring PTSD and substance use disorders. American Journal of Psychiatry, 167, 11-13. doi:10.1176/appi.ajp.2009.09111602

Back, S. E., Dansky, B. S., Carroll, K. M., Foa, E. B., \& Brady, K. T. (2001). Exposure therapy in the treatment of PTSD among cocaine-dependent individuals: Description of procedures. Journal of Substance Abuse Treatment, 21, 35-45. doi:10.1016/S0740$5472(01) 00181-7$

Back, S., Waldrop, A., Hien, D., \& Brady, K. (2006). Evidenced-based time-limited treatment of co-occurring substance use disorders and civilian-related PTSD. Brief Treatment and Crisis Intervention, 6, 283-294. doi:10.1093/brief-treatment/mhl013 
Becker, C., Zayfert, C., \& Anderson, E. (2004). A survey of psychologists' attitudes towards and utilization of exposure therapy for PTSD. Behavior Research and Therapy, 42, 277-292. doi:10.1016/S0005-7967(03)00138-4

Blake, D., Weathers, F., Nagy, L., Kaloupek, D., Gusman, F., Charney, D., \& Keane, T. (1995). The development of a clinician-administered PTSD scale. Journal of Traumatic Stress, 8 , 75-90. doi:10.1007/BF02105408

Bonn-Miller, M. O., Vujanovic, A. A., Boden, M. T., \& Gross, J. J. (2011). Posttraumatic stress, difficulties in emotion regulation, and coping-oriented marijuana use. Cognitive Behaviour Therapy, 40, 34-44. doi:10.1080/16506073.2010.525253

Boucsein, W. (1992). Electrodermal activity. New York: Plenum Press

Brady, K., Dansky, B., Back, S., Foa, E. \& Carroll, K. M. (2001). Exposure therapy in the treatment of PTSD among cocaine-dependent individuals: Preliminary findings. Journal of Substance Abuse Treatment, 21, 47-54. doi:10.1016/S0740-5472(01)00182-9

Brady, K., Killeen, T., Saladen, M., Dansky, B., \& Becker, S. (1994). Comorbid substance abuse and posttraumatic stress disorder: Characteristics of women in treatment. American Journal on Addictions, 3, 160-163. doi:10.3109/10550499409117249

Bradley, B., DeFife, J. A., Guarnaccia, C., Phifer, J., Fani, N., Ressler, K. J., \& Westen, D. (2011). Emotion dysregulation and negative affect: association with psychiatric symptoms. Journal of Clinical Psychiatry, 72, 685-691. doi:10.4088/JCP.10m06409blu

Bradley, M., Miccoli, L., Escrig, M. \& Lang, P. (2008). The pupil as a measure of emotional arousal and autonomic activation. Psychophysiology, 45, 602-607.

Braithwaite, J. J., Watson, D. G., Jones, R., \& Rowe, M. (2013). A guide for analyzing electrodermal activity (EDA) \& skin conductance responses (SCRs) for psychological 
experiments. Retrieved from University of Birmingham website:

http://www.birmingham.ac.uk/documents/college-les/psych/saal/guide-electrodermalactivity.pdf

Briere, J., Hodge, M., \& Godbout, N. (2010). Traumatic stress, affect dysregulation and dysfunctional avoidance: A structural equation model. Journal of Traumatic Stress, 23, 767-774. doi:10.1002/jts.20578

Calkins, S. D. (1994). Origins and outcomes of individual differences in emotion regulation. Monographs of the Society for Research in Child Development, 59, 53-72. doi:10.2307/1166138

Carroll, K. M. (1996). Relapse prevention as a psychosocial treatment: A review of controlled clinical trials. Experimental and Clinical Psychopharmacology, 4, 46-54. doi:10.1037/1064-1297.4.1.46

Carroll, K. M., Nich, C., \& Rounsaville, B. J. (1998). Utility of therapist session checklists to monitor delivery of coping skills treatment for cocaine abusers. Psychotherapy Research, 8, 307-320. doi:10.1093/ptr/8.3.307

Carroll, K. M., Rounsaville, B. J., \& Gawin, F. H. (1991). A comparative trial of psychotherapies for ambulatory cocaine abusers: Relapse prevention and interpersonal psychotherapy. American Journal of Drug and Alcohol Abuse, 17, 229-247. doi:10.3109/00952999109027549

Cheetham, A., Allen, N., Yücel, M., \& Lubman, D. (2010). The role of affective dysregulation in drug addiction. Clinical Psychology Review, 30, 621-634. doi:10.1016/j.cpr.2010.04.005 
Chilcoat, H. D., \& Breslau, N. (1998). Investigations of causal pathways between PTSD and drug use disorders. Addictive Behaviors, 23, 827-840. doi:10.1016/S03064603(98)00069-0

Chilcoat, H. D., \& Menard, C. (2003). Epidemiological investigations: Comorbidity of posttraumatic stress disorder and substance use disorder. In P. Ouimette \& P. J. Brown (Eds.), Trauma and substance abuse: Causes, consequences, and treatment of comorbid disorders (pp. 9-28). Washington, DC: American Psychological Association.

Childress, A. R., Ehrman, R., McLellan, A. T., MacRae, J., Natale, M., \& O’Brien, C. P. (1994). Can induced moods trigger drug-related responses in opiate abuse patients? Journal of Substance Abuse Treatment, 11, 17-23. doi:10.1016/0740-5472(94)90060-4

Cicchetti, D., Ganiban, J., \& Barnett, D. (1991). Contributions from the study of high-risk populations to understanding the development of emotion regulation. In J. Garber \& K. Dodge (Eds.), The development of emotion regulation and dysregulation (pp. 15-49). New York, NY: Cambridge University Press.

Coffey, S. F., Stasiewicz, P. R., Hughes, P. M., \& Brimo, M. L. (2006). Trauma-focused imaginal exposure for individuals with comorbid posttraumatic stress disorder and alcohol dependence: Revealing mechanisms of alcohol craving in a cue reactivity paradigm. Psychology of Addictive Behaviors, 20, 425-435. doi:10.1037/0893164X.20.4.425

Cole, P., Martin, S., \& Dennis, T. (2004). Emotion regulation as a scientific construct: methodological challenges and directions for child development research. Child Development, 75, 317-333. doi:10.1111/j.1467-8624.2004.00673.x 
Cole, P., Michel, M., \& Teti, L. (1994). The development of emotion regulation and dysregulation: a clinical perspective. Monograph of Social Research and Child Development, 59, 73-100. doi:10.2307/1166139

Cooper, M. L., Frone, M. R., Russell, M., \& Mudar, P. (1995). Drinking to regulate positive and negative emotions: A motivational model of alcohol use. Journal of Personality and Social Psychology, 69, 990-1005. doi:10.1037//0022-3514.69.5.990

Dawson, M. E., Schell, A. M., \& Filion, D. L. (2007). The electrodermal system. In J. Cacioppo, L. Tassinary, \& G. Berntson (Eds.), Handbook of psychophysiology (pp. 159-181). New York, NY: Cambridge University Press.

Donovan, B., Padin-Rivera, E., \& Kowaliw S. (2001). “Transcend”: initial outcomes from a posttraumatic stress disorder/substance abuse treatment program. Journal of Trauma Stress, 14,757-772. doi:10.1023/A:1013094206154

Duncan, D. F. (1975). The acquisition, maintenance and treatment of polydrug dependence: A public health model. Journal of Psychedelic Drugs, 7, 201-213. doi:10.1080/02791072.1975.10472000

Eisenberg, N., Cumberland, A., \& Spinrad, T. L. (1998). Parental socialization of emotion. Psychological Inquiry, 9, 241-273.

Etkin, A., Egner, T., \& Kalisch, R. (2011). Emotional processing in anterior cingulate and medial prefrontal cortex. Trends in Cognitive Science, 15, 85-93. doi:10.1016/j.tics.2010.11.004

Foa, E., Keane, T. M., Friedman, M. J., \& Cohen, J. A. (2008). Effective treatments for PTSD: practice guidelines from the International Society for Traumatic Stress Studies. New York, NY: Guilford. 
Foa, E., \& Kozak, M. (1986). Emotional processing of fear: Exposure to corrective information. Psychological Bulletin, 99, 20-35. doi:10.1037//0033-2909.99.1.20

Foa, E., \& Rothbaum, B. O. (1998). Treating the trauma of rape: Cognitive-behavioral therapy for PTSD. New York, NY: Guilford.

Foa, E., \& Tolin, D. (2000). Comparison of the PTSD Symptom Scale-Interview Version and the Clinician-Administered PTSD scale. Journal of Traumatic Stress, 13, 181-191. doi:10.1023/A:1007781909213

Fonagy, P., Gergely, G., Jurist, E. L., \& Target, M. (2002). Affect regulation, mentalization and the development of the self. New York, NY: Other Press.

Ford, J. D., Hawke, J., Alessi, S., Ledgerwood, D., \& Petry, N. (2007). Psychological trauma and PTSD symptoms as predictors of substance dependence treatment outcomes. Behaviour Research and Therapy, 45, 2417-2431. doi:10.1016/j.brat.2007.04.001

Ford, J. D., Russo, E. M., \& Mallon, S. D. (2007). Integrating treatment of posttraumatic stress disorder and substance use disorder. Journal of Counseling and Development, 4, 475490. doi:10.1002/j.1556-6678.2007.tb00616.x

Glover, E. (1932). On the aetiology of drug addiction. International Journal of Psychoanalysis, 13, 298-328. Retrieved from http://www.psychoanalysis.org.uk/ijpa/

Gratz, K. L., \& Roemer, L. (2004). Multidimensional assessment of emotion regulation and dysregulation: Development, factor structure, and initial validation of the difficulties in emotion regulation scale. Journal of Psychopathology and Behavioral Assessment, 26, 41-54. doi:10.1023/B:JOBA.0000007455.08539.94

Gross, J. J. (2002). Emotion regulation: Affective, cognitive and social consequences. Psychopathology, 39, 281-291. 
Gross, J. J., \& Munoz, R. F. (1995). Emotion regulation and mental health. Clinical Psychology: Science and Practice, 2, 151-164. doi:10.1111/j.1468-2850.1995.tb00036.x

Gross, J. J., \& Thompson, R. A. (2007). Emotion regulation: Conceptual foundations. In J. J. Gross (Ed.), Handbook of emotion regulation (pp. 3-24). New York, NY: Guilford.

Gunnar, M. R., \& Fisher P. A. (2006). The early experience, stress and prevention: Bringing basic research on early experience and stress neurobiology to bear on preventive intervention research on neglected and maltreated children. Developmental Psychopathology, 18, 651-677. doi:10.1017/S0954579406060330

Hagenaars, M. A., van Minnen, A., \& Hoogduin, C. A. L. (2010). The impact of dissociation and depression on the efficacy of exposure treatment for PTSD. Behaviour Research and Therapy, 48, 19-27. doi:10.1016/j.brat.2009.09.001

Hamburg, M., \& Collins, F. (2010). The path to personalized medicine. The New England Journal of Medicine, 363, 301-304.

Harrington, T., \& Newman, E. (2007). The psychometric utility of two self-report measures of PTSD among women substance users. Addictive Behaviors, 32, 2788-2798. doi:10.1016/j.addbeh.2007.04.016

Hayes, S. C., Wilson, K. G., Gifford, E. V., Follette, V. M., \& Strosahl, K. (1996). Experiential avoidance and behavioral disorders: A functional dimensional approach to diagnosis and treatment. Journal of Consulting and Clinical Psychology, 64, 1152-1168. doi:10.1037//0022-006X.64.6.1152

Herman, J., Ostrander, M., Mueller, N., \& Figueiredo, H. (2005). Limbic system mechanisms of stress regulation: Hypothalamo-pituitary-adrenocortical axis Progress. Progress in Neuro-Psychopharmacology \& Biological Psychiatry, 29, 1201-1213. Retrieved from 
http://www.journals.elsevier.com/progress-in-neuro-psychopharmacology-andbiological-psychiatry/

Hertel, J., Schutz, A., \& Lammers, C. H. (2009). Emotional intelligence and mental disorder. Journal of Clinical Psychology, 65, 942-954. doi:10.1002/jclp.20597

Hien, D. A., Campbell, A. N. C., Ruglass, L. M., Hu, M., \& Killeen, T. (2010). The role of alcohol misuse in PTSD outcomes for women in community treatment: A secondary analysis of NIDA's Women and Trauma Study. Drug and Alcohol Dependence, 111, 114-119. doi:10.1016/j.drugalcdep.2010.04.011

Hien, D. A., Cohen, L. R., \& Campbell, A. N. C. (2005). Is traumatic stress a vulnerability factor for development of substance use disorders? Clinical Psychology Review, 25, 813-823. doi:10.1016/j.cpr.2005.05.006

Hien, D. A., Cohen, L. R., Litt, L., Miele, G. M., \& Capstick, C. (2004). Promising empirically supported treatments for substance-using women with PTSD. American Journal of Psychiatry, 161, 1426-1432. doi:10.1176/appi.ajp.161.8.1426

Hien, D. A., Jiang, H., Campbell, A. N. C., Hu, M., Miele, G. M., Cohen, L. R., . . Nunes, E. V. (2010). Do treatment improvements in PTSD severity affect substance use outcomes? A secondary analysis from a randomized clinical trial in NIDA's clinical trials network. American Journal of Psychiatry, 167, 95-101. doi:10.1176/appi.ajp.2009.09091261

Hien, D. A., Litt, L. C., Cohen, L. R., Miele, G. M., \& Campbell, A. N. C. (2009). Trauma services for women in substance abuse treatment: An integrated approach. Washington, DC: American Psychological Association. 
Hien, D. A., \& Miele G. M. (2003). Emotion-focused coping as a mediator of maternal cocaine abuse and antisocial behavior. Psychology of Addictive Behavior, 17, 49-55. doi:10.1037/0893-164X.17.1.49

Hien, D. A., Nunes, E. V., Levin, F. R., \& Fraser, D. (2000). Posttraumatic stress disorder and short-term outcome in early methadone treatment. Journal of Substance Abuse Treatment, 19, 31-37. doi:10.1016/S0740-5472(99)00088-4

Hien, D. A., \& Zimberg, S. (1991). Demographic and Treatment History Form [Measurement instrument]. New York, NY: St. Lukes/Roosevelt Hospital Center.

Horowitz, H. A., Overton, W. F., Rosenstein, D., \& Steidl, J. H. (1992). Comorbid adolescent substance abuse: A maladaptive pattern on self-regulation. Adolescent Psychiatry: Developmental and Clinical Studies, 18, 465-483. Retrieved from http://benthamscience.com/journal/index.php?journalID=aps

Kalisch, R. (2009). The functional neuroanatomy of reappraisal: Time matters. Neuroscience and Biobehavior Review, 33, 1215-1226. doi:10.1016/j.neubiorev.2009.06.003

Kanske, P., Heissler, J., Schönfelder, S., \& Wessa, M. (2012). Neural correlates of emotion regulation deficits in remitted depression: the influence of regulation strategy, habitual regulation use, and emotional valence. Neuroimage, 61, 686-693. doi:10.1016/j.neuroimage.2012.03.089

Kessler, R. C., Sonnega, A., Bromet, E., Hughes, M., \& Nelson, C. B. (1995). Posttraumatic stress disorder in the National Comorbidity Survey. Archives of General Psychiatry, 52, 1048-1060. doi:10.1001/archpsyc.1995.03950240066012 
Khantzian, E. J. (1997). The self-medication hypothesis of substance use disorders: A reconsideration and recent applications. Harvard Review of Psychiatry, 4, 231-244. doi:10.3109/10673229709030550

Khantzian, E. J. (2003). The self-medication hypothesis revisited: The dually diagnosed patient. Primary Psychiatry, 10, 47-48, 53-54. Retrieved from http://primarypsychiatry.com/

Killeen, T. K., Back, S. E., \& Brady, K. T. (2011). The use of exposure-based treatment among individuals with PTSD and co-occurring substance use disorders: Clinical considerations. Journal of Dual Diagnosis, 7, 194-206. doi:10.1080/15504263.2011.620421

Koob, G. F. (2003). Alcoholism: Allostasis and beyond. Alcoholism: Clinical and Experimental Research, 27, 232-243. doi:10.1097/01.ALC.0000057122.36127.C2

Krystal, H., \& Raskin, H. A. (1970). Drug dependence: Aspects of ego functions. Detroit: Wayne State University Press.

Main, M., \& Solomon, J. (1990). Procedures for identifying infants as disorganized/disoriented during the Ainsworth Strange Situation. In M. T. Greenberg, D. Cicchetti, \& E. M. Cummings (Eds.), Attachment during the preschool years: Theory, research and intervention (pp. 121-160). Chicago, IL: University of Chicago Press.

Marlatt, A. G., \& Gordon, J. R. (1985). Relapse prevention: Maintenance and strategies in the treatment of addictive behaviours. New York, NY: Guilford.

McDermott, M. J., Tull, M. T., Gratz, K. L., Daughters, S. B., Lejuez, C. W. (2009). The role of anxiety sensitivity and difficulties in emotion regulation in posttraumatic stress disorder among crack/cocaine dependent patients in residential substance abuse treatment. Journal of Anxiety Disorders, 23, 591-599. doi:10.1016/j.janxdis.2009.01.006 
McGovern, M. P., Lambert-Harris, C., Acquilano, S., Xie, H., Alterman, A. I., \& Weiss, R. D. (2009). A cognitive behavioral therapy for co-occurring substance use and posttraumatic stress disorders. Addictive Behaviors, 34, 892-897. doi:10.1016/j.addbeh.2009.03.009

McRae, K., Ochsner, K. N., Mauss, I. B., Gabrieli, J. D. E., \& Gross, J. J. (2008). Gender differences in emotion regulation: An fMRI study of cognitive reappraisal. Group Processes and Intergroup Relations, 11, 143-162. doi:10.1177/1368430207088035

Mills, K., Teesson, M., Back, S., Brady, K., Baker, A., Hopwood, S., . . Ewer, P. (2012). Integrated exposure-based therapy for co-occuring posttraumatic stress disorder and substance dependence: A randomized controlled trial. Journal of the American Medical Association, 308, 690-699. doi:10.1001/jama.2012.9071

Najavits, L. M. (2002). Seeking Safety: Cognitive-behavioral therapy for PTSD and substance abuse. New York, NY: Guilford.

Najavits, L. M, Schmitz, S., Gotthardt, S., \& Weiss, R. D. (2005). Seeking Safety plus exposure therapy for dual diagnosis men. Journal of Psychoactive Drugs, 27, 425-435. Retrieved from http://www.journalofpsychoactivedrugs.com/index.html

Najavits, L. M., Harned, M., Gallop, R., Butler, S., Barber, J., Thase, M., \& Crits-Christoph, P. (2007). Six-month treatment outcomes of cocaine-dependent patients with and without PTSD in a multisite national trial. Journal of Studies on Alcohol and Drugs, 68, 353361. Retrieved from http://www.jsad.com/

Nolen-Hoeksema, S., Wisco, B., \& Lyubomirsky, S. (2008). Rethinking rumination. Perspectives on Psychological Science, 3, 400-424. doi:10.1111/j.17456924.2008.00088.x 
Ochsner, K., \& Gross, J. (2005). The cognitive control of emotion. Trends Cognitive Science, 9 , 242-249. doi:10.1016/j.tics.2005.03.010

Ouimette, P., Goodwin, E., \& Brown, P. J. (2006). Health and well being of substance use disorder patients with and without posttraumatic stress disorder. Addictive Behaviors, 31, 1415-1423. doi:10.1016/j.addbeh.2005.11.010

Pearlman, L. A., \& Courtois, C. A. (2005). Clinical applications of the attachment framework: Relational treatment of complex trauma. Journal of Traumatic Stress, 18, 449-459. doi: $10.1002 /$ jts.20052

Pietrzak, R. H., Goldstein, R. B., Southwick, S. M., \& Grant, B. F. (2011). Medical comorbidity of full and partial posttraumatic stress disorder in U.S. adults: Results from Wave 2 of the National Epidemiologic Survey on Alcohol and Related Conditions. Psychosomatic Medicine, 73, 697-707. doi:10.1097/PSY.0b013e3182303775

Pitman, R. K., Altman, B., Greenwald, E., Longpre, R. E., Macklin, M. L., Poire, R. E., \& Steketee, G. S. (1991). Psychiatric complications during flooding therapy for posttraumatic stress disorder. Journal of Clinical Psychiatry, 52, 17-20. Retrieved from http://www.psychiatrist.com/Pages/home.aspx

Radó, S. (1933). Psychoanalysis of pharmacothymia. Psychoanalytic Quarterly, 2, 1-23. Retrieved from http://onlinelibrary.wiley.com/journal/10.1002/(ISSN)2167-4086

Ressler, K. J., Rothbaum, B. O., Tannenbaum, L., Anderson, P., Graap, K., Zimand, E., . . Davis, M. (2004). Cognitive enhancers as adjuncts to psychotherapy: Use of Dcycloserine in phobic individuals to facilitate the extinction of fear. Archives of General Psychiatry, 61, 1136-1144. Retrieved from http://archpsyc.jamanetwork.com /journal.aspx 
Rawson, R. A., Obert, J. L., McCann, M. J., \& Marinelli-Casey, P. J. (1993). Relapse prevention strategies in outpatient substance abuse treatment. Psychology of Addictive Behaviors, 7, 85-95. doi:10.1037/0893-164X.7.2.85

Savov, S., \& Atanassov, N. (2013). Deficits of affect mentalization in patients with drug addiction: Theoretical and clinical aspects. Addiction, 2013(250751). doi: $10.1155 / 2013 / 250751$

Schore, A. (1994). Affect regulation and the origin of the self: The neurobiology of emotional development. Mahwah, NJ: Erlbaum.

Schore, A. (2003). Affect dysregulation and disorders of the self. New York, NY: Norton.

Sloan, D., \& Kring, A. M. (2007). Measuring changes in emotion during psychotherapy: Conceptual and methodological issues. Clinical Psychology: Science and Practice, 14, 307-322. doi:10.1111/j.1468-2850.2007.00092.x

Snyder, J., Schrepferman, L., \& St. Peter, C. (1997). Origins of antisocial behavior: Negative reinforcement and affect dysregulation of behavior as socialization mechanisms in family interaction. Behavior Modification, 21, 187-215. doi:10.1177/01454455970212004

Sobell, L. C., \& Sobell, M. B. (1992). Timeline follow back: A technique for assessing selfreported alcohol consumption. In R. Z. Litten, \& J. Allen (Eds.), Measuring alcohol consumption: Psychosocial and biological methods (pp. 41-72). Clifton, NJ: Humana Press.

Sobell, L. C., \& Sobell, M. B. (1995). Alcohol consumption measures. In J. P. Allen \& M. Columbus (Eds.), Assessing alcohol problems: A guide for clinicians and researchers (pp. 55-73). Rockville, MD: National Institute on Alcohol Abuse and Alcoholism. 
Sobell, L. C., \& Sobell, M. B. (2000). Timeline Followback (TLFB). In Handbook of psychiatric measures (pp. 477-479). Washington, DC: American Psychiatric Association.

Southwick, S., Ozbay, F., Charney, D., \& McEwen, B. (2008). Adaptation to stress and psychobiological mechanisms of resilience. In B. Lukey \& V. Tepe (Eds.), Biobehavioral resilience to stress (pp. 91-114). Boca Raton: CRC Press.

Stewart, S. H., \& Conrod, P. J. (2002). Psychosocial models of functional associations between posttraumatic stress disorder and substance use disorder. In P. C. Ouimette \& P. J. Brown (Eds.), Trauma and substance abuse: Causes, consequences, and treatment of comorbid disorders (pp. 29-55). Washington, DC: American Psychological Association.

Storm, H. (2008). Changes in skin conductance as a tool to monitor nociceptive stimulation and pain. Current Opinion in Anesthesiology, 21, 796-804.

doi:10.1097/ACO.0b013e3283183fe4

Storm, H., Myre, K., Rostrup, M., Stokland, O., Lien, M. D., \& Raeder, J. C. (2002). Skin conductance correlates with perioperative stress. Acta Anaesthesiologica Scandinavica, 46, 887-895. doi:10.1034/j.1399-6576.2002.460721.x

Suh, J. J., Ruffins, S., Robins, C. E., Albanese, M. J., \& Khantzian, E. J. (2008). Self-medication hypothesis. Psychoanalytic Psychology, 25, 518-532. doi:10.1037/0736-9735.25.3.518

Tamir, M., Robinson, M. D., \& Clore, G. L. (2002). The epistemic benefits of trait-consistent mood states: An analysis of extraversion and mood. Journal of Personality and Social Psychology, 83, 663-677. doi:10.1037//0022-3514.83.3.663

Teicher, M.H. (2000). Wounds that won't heal: The neurobiology of child abuse. Cerebrum, 2(4), 50-62. Retrieved from http://dana.org/cerebrum/ 
Teicher, M. H. (2002, March). Scars that won't heal: The neurobiology of child abuse. Scientific American. Retrieved from http://www.scientificamerican.com/

Thayer, J., Ahs, F., Fredrickson, M., Stollers, J., Wager, J. (2012). A meta-analysis of heart rate variability and neuroimaging studies: Implications for heart rate variability as a marker of stress and health. Neuroscience Behavior Reviews, 36, 747-756.

doi:10.1016/j.neubiorev.2011.11.009

Thompson, R. A. (1991). Emotional regulation and emotional development. Educational Psychology Review, 3, 269-307. doi:10.1007/BF01319934

Thorberg, F. A., \& Lyvers, M. (2006). Negative mood regulation (NMR) expectancies, mood, and affect intensity among clients in substance disorder treatment facilities. Addictive Behaviors, 31, 811-820. doi:10.1016/j.addbeh.2005.06.008

Triffleman, E., Carroll, K., \& Kellogg, S. (1999). Substance dependence posttraumatic stress disorder therapy: An integrated cognitive-behavioral approach. Journal of Substance Abuse Treatment, 17, 3-14. doi:10.1016/S0740-5472(98)00067-1

Tronick, E. (1989). Emotions and emotional communication in infants. American Psychologist, 44, 112-119. doi:10.1037//0003-066X.44.2.112

Tull, M. T., Barrett, H. M., McMillan, E. S., \& Roemer, L. (2007). A preliminary investigation of the relationship between emotion regulation difficulties and posttraumatic stress symptoms. Behavior Therapy, 38, 303-313. doi:10.1016/j.beth.2006.10.001

van Dam, D., Ehring, T., Vedel, E., \& Emmelkamp, P. M. (2010). Screening for posttraumatic stress disorder in civilian substance use disorder patients: Cross-validation of the Jellinek-PTSD screening questionnaire. Journal of Substance Abuse Treatment, 44, 12631. doi:10.1016/j.jsat.2012.03.005 
van Dam, D., Vedel, E., Ehring, T., Emmelkamp, P. M., (2012). Psychological treatments for concurrent posttraumatic stress disorder and substance use disorder: A systematic review. Clinical Psychology Review, 32, 202-214. doi:10.1016/j.cpr.2012.01.004

van der Kolk, B. A., Pelcovitz, D., Roth, S., Mandel, F. S., McFarlane, A., \& Herman, J. L. (1996). Dissociation, somatization, and affect dysregulation: The complexity of adaptation to trauma. American Journal of Psychiatry, 153, 83-93. Retrieved from http://ajp.psychiatryonline.org/journal.aspx?journalid=13

van Minnen, A., Harned, M. S, Zoellner, L., \& Mills, K. (2012). Examining potential contraindications for prolonged exposure therapy for PTSD. European Journal of Psychotraumatology, 3(18805). doi:10.3402/ejpt.v3i0.18805

Weinberg, A., \& Klonsky, E. D. (2009). Measurement of emotion dysregulation in adolescents. Psychological Assessment, 21, 616-621. doi:10.1037/a0016669

Weiss, N., Tull, M., Anestis, M., \& Gratz K. (2013). The relative and unique contributions of emotion dysregulation and impulsivity to posttraumatic stress disorder among substance dependent inpatients. Drug and Alcohol Dependence, 128, 45-51.

doi:10.1016/j.drugalcdep.2012.07.017

Zlomke, K. R., \& Hahn, K. S. (2010). Cognitive emotion regulation strategies: gender differences and associations to worry. Personality \& Individual Differences, 48, 408-413. doi:10.1016/j.paid.2009.11.007 